\title{
Design and Preliminary Evaluation of a Wearable Passive Cam-Based Shoulder Exoskeleton
}

\section{Morteza Asgari ( $\square$ sasgari@vols.utk.edu )}

The University of Tennessee Knoxville https://orcid.org/0000-0003-4179-7055

\section{Elizabeth A. Phillips}

The University of Tennessee Medical Center

\section{Britt M. Dalton}

The University of Tennessee Medical Center

Jennifer L. Rudl

The University of Tennessee Medical Center

Dustin L. Crouch

The University of Tennessee Knoxville

\section{Research}

Keywords: Assistive technology, Exoskeletons, Human-robot interaction, Shoulder disability

Posted Date: April 5th, 2021

DOl: https://doi.org/10.21203/rs.3.rs-355805/v1

License: (c) (1) This work is licensed under a Creative Commons Attribution 4.0 International License. Read Full License 
Title:

Authors:

Morteza Asgari, M.Sc., Department of Mechanical, Aerospace, and Biomedical Engineering, University of Tennessee, Knoxville.

10 Elizabeth A. Phillips, PT, DPT, GCS, Brain and Spine Institute, Department of Rehabilitation Services, University of Tennessee 11 Medical Center, Knoxville

12 Britt M. Dalton, PT, DPT, OCS, COMT, FAAOMPT, Brain and Spine Institute, Department of Rehabilitation Services, 13 University of Tennessee Medical Center, Knoxville

14 Jennifer L. Rudl, OTR/L, Brain and Spine Institute, Department of Rehabilitation Services, University of Tennessee Medical 15 Center, Knoxville

16 Dustin L. Crouch, Ph.D., Department of Mechanical, Aerospace, and Biomedical Engineering, University of Tennessee, 17 Knoxville.

Corresponding Author:

21 Morteza Asgari (e-mail: sasgari@ vols.utk.edu) 


\section{Exoskeleton}

Abstract

Background: Mechanically passive (i.e. spring-powered) exoskeletons may be a practical and affordable solution to meet a growing clinical need for continuous, home-based movement assistance. We designed, fabricated, and preliminarily evaluated the performance of a wearable, passive, cam-driven shoulder exoskeleton (WPCSE) prototype.

Methods: The novel feature of the WPCSE is a modular spring-cam-wheel module, which generates an assistive force that can be customized to compensate for any proportion of the shoulder elevation moment due to gravity. We performed a benchtop experiment to validate the mechanical output of the WPCSE against our theoretical model. We also conducted a pilot biomechanics study (four able-bodied subjects) to quantify the effect of a WPCSE prototype on muscle activity and shoulder kinematics during three one-degree-of-freedom shoulder movements.

Results: The shoulder elevation moment produced by the spring-cam-wheel module alone closely matched the desired, theoretical moment. However, when measured from the full WPCSE prototype, the moment was lower (up to 30\%) during positive shoulder elevation and higher (up to 120\%) during negative shoulder elevation compared to the theoretical moment, due primarily to friction. Even so, a WPCSE prototype, compensating for about $25 \%$ of the shoulder elevation moment due to gravity, showed a trend of reducing root mean square (up to 50\%) and peak (up to 53\%) electromyogram magnitudes of several muscles crossing the shoulder during shoulder elevation and horizontal adduction/abduction movements. Subjects verbally reported that the WPCSE did not physically constrain them during the tested movements.

Conclusion: The results provide proof-of-concept evidence that our WPCSE can potentially assist shoulder movements. The proposed WPCSE, once refined, could provide clinical and home-based rehabilitation for patients with shoulder disability. 


\section{Introduction}

Shoulder disability is a global health burden $[1,2]$ associated with several common orthopedic and neurological disorders, such as rotator cuff tear [3], peripheral nerve injury [4], muscle atrophy [5], and stroke [6]. Many people with shoulder disability have mild to moderate disability, meaning that they can still move their shoulder but with lower strength and range of motion than that of a healthy shoulder. Furthermore, people with shoulder disability often have trouble elevating the shoulder or holding the arm up against gravity. Such functional deficits can make it hard for individuals to perform activities of daily living, particularly those for which gravity is a dominant contributor to shoulder loading [7]. Consequently, shoulder disability can degrade a person's quality of life, self-esteem, and independence.

For people with shoulder disability, there is a clinical need for medical devices that can provide continuous, home-based movement assistance to compensate for gravity at the shoulder. Gravity compensation can assist upper extremity motor function 11 by reducing the joint moments that muscles need to generate for a given movement [8]. We previously showed that anti-gravity assistance reduces activations of muscles that mainly contribute to positive shoulder elevation [9]. By reducing muscle activations,

13 gravity compensation at the shoulder could help people perform upper extremity tasks with less effort and reduce muscle tissue 14 loads. Additionally, for people with shoulder disability, gravity compensation could enhance range of motion and shoulder strength by encouraging them to be more physically active.
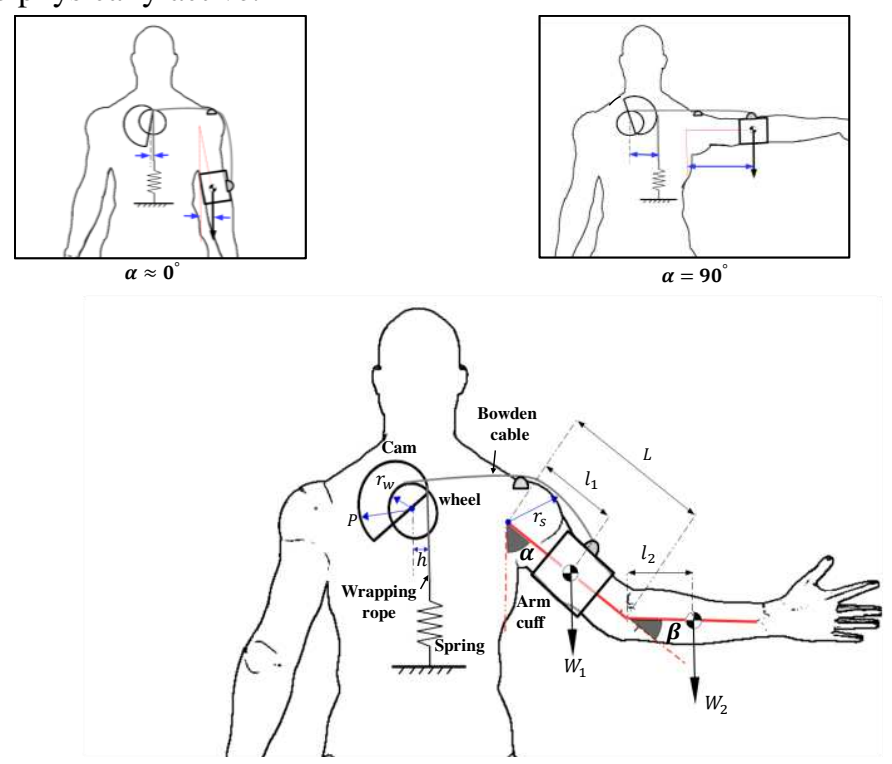

Fig 1. Schematic diagram of shoulder exoskeleton system concept (posterior view on right arm). Upper left and right figures represent two extreme configurations for shoulder elevation in the frontal plane. As the shoulder elevates from the $0^{\circ}$ (upper left figure) to $90^{\circ}$ (upper right figure), the cam-wheel rotates and adjust its effective moment arm relative to the wrapping rope to modulate the Bowden cable pulling force. 
A wide variety of assistive and rehabilitative exoskeletons (sometimes called orthoses) have been developed for people with disability. Exoskeletons, which are typically worn on and transmit forces to the body [10], generate forces either actively or passively. Active exoskeletons, such as ARMin III [11] and MGA [12] use powered actuators, usually located near the joints, to generate forces that are transmitted to the body by rigid links. The high weight and rotational inertia reduce the transparency and power density of these exoskeletons. Some powered exoskeletons incorporate cable-driven mechanisms, which increases the power-to-weight ratio by allowing actuators to be mounted farther away from the user's joints. Cable-driven exoskeletons can have either serial or parallel design architecture. Serial cable-driven exoskeletons such as CADEN-7 [10], L-EXOS [13], and IntelliArm [14] still use rigid links with revolute joints but have lower weight and rotational inertia compared to conventional exoskeletons. can provide more flexible assistance since the forces they generate can be modulated by control software. However, they are settings. Conversely, parallel cable-driven systems such as CAREX [15] and CARR-4 [16] use flexible cables with rigid joint cuffs to actuate the exoskeleton, which highly reduces the overall weight and moment of inertia of the moving parts. Active exoskeletons relatively heavy, large, expensive, and complex because they require motors, power sources (e.g. batteries), and computer hardware. These features make active exoskeletons less wearable and portable, limiting their application to clinical and laboratory settings.

Passive exoskeletons generate assistive forces using counterweights (such as the Mobility Arm, Nitzbon), rubber bands [17], or springs [18]. The assistive forces generated by passive exoskeletons are fixed according to their mechanical design and cannot be modulated in real time, as with active exoskeletons. However, passive exoskeletons are appealing for providing continuous, homebased movement assistance since they do not require electromechanical hardware (e.g. motors, batteries). Thus, passive exoskeletons are potentially more lightweight, cost-effective, low-maintenance, wearable, and portable than active exoskeletons.

Existing wearable passive shoulder exoskeletons compensate for gravity by releasing and storing energy from elastic springs as the shoulder is elevated and lowered, respectively [19]. Commercially available wearable passive shoulder exoskeletons, intended primarily for occupational or industrial settings, include EksoVest (Exo Bionics Holdings, Inc.), SuitX (US Bionics, Inc.), and AirFrame (Levitate technologies, Inc.). However, these existing exoskeletons may not be ideal for assisting activities of daily living for people with shoulder disability because they are designed for application-specific and high-load tasks (e.g. overhead drilling and heavy lifting) [20, 21]. They also typically have a high profile (i.e. they extend out far from the user's body) because they incorporate rigid linkages and mechanical joints.

There are several passive devices that have been developed and commercialized for clinical applications. Early devices include 
passive mobile arm supports, such as SaeboMAS, that mount on a wheelchair, wall, or table [22, 23] and compensate for gravity using counterweights [24] or parallelograms that are equipped with spring-cam [18] or spring mechanisms. The Wilmington Robotic Exoskeleton (WREX) [17], is another wheelchair-mounted arm support that uses elastic bands to compensate for decoupled gravity at the shoulder and elbow joints. T-WREX, a more recent version of the WREX [19, 25], was equipped with position and grip sensors that permit movement therapy through virtual interfaces (e.g. computer games). These passive devices can completely balance the arm and are mostly intended for patients who have severe neurological shoulder disability. However, their level of gravity compensation is usually unmodulated over the shoulder's range of motion, and they must be mounted on rigid frames or fixed bases. These features make such devices an inappropriate solution for patients with greater mobility and only mild to moderate shoulder disability.

We propose a new wearable, passive, cam-based shoulder exoskeleton (WPCSE) that addresses limitations of existing devices for providing continuous, at-home assistance to patients with mild to moderate shoulder disability. The main, novel contribution of the WPCSE is a spring-cam-wheel module whose assistive force can be customized for each patient to compensate for any proportion of the shoulder elevation moment due to gravity. Additionally, the assistive force is transmitted to the arm through a Bowden cable that passes through self-aligning spherical bearings, which allows the WPCSE to assist movements across a wide range of shoulder elevation planes. Since the spring-cam-wheel module is compact and lightweight, the WPCSE could potentially be worn on the body comfortably throughout the day under clothing. The overall objective of our study was to design, fabricate, and preliminarily evaluate a WPCSE prototype that includes the novel spring-cam-wheel mechanism. In Methods section, we first present a detailed description of the WPCSE theoretical model and prototype design. We then developed a physical benchtop model to quantify the mechanical performance of the spring-cam-wheel module and WPCSE. We expected that friction and spring hysteresis, which were unaccounted for in our theoretical model, would reduce the assistive moment generated by the physical benchtop model compared to the theoretical model. Finally, we performed a pilot biomechanics study to preliminarily test the concept that the WPCSE could reduce the activity of muscles crossing the shoulder; secondarily, we evaluated the effect of the WPCSE on shoulder kinematics. The pilot study provided data needed for a power analysis to design a future, more comprehensive biomechanical study to validate the efficacy of the WPCSE.

\section{Methods}


Our WPCSE compensates for gravity at the shoulder using an elastic spring whose force is transmitted to the arm through a Bowden cable that passes over the superior aspect of the shoulder (Fig. 1). A common example of a gravity compensation system is the use of counterweights to balance the weight and reduce the force required to raise and lower an elevator car. Instead of counterweights, our exoskeleton uses a spring that is stretched when the arm is beside the body and pulls on the Bowden cable as the arm elevates.

As the shoulder elevation angle increases from $0^{\circ}$ to $90^{\circ}$, the magnitude of the negative shoulder elevation moment due to gravity increases. We designed our exoskeleton to provide increasing assistance in proportion to the increasing gravity moment. This was accomplished by incorporating a cam-wheel component between the spring and arm (Fig. 1). The variable-radius cam and a constant-radius wheel are fixed to each other so that they rotate together about the same axis. As shoulder elevation angle increases, the moment arm of the of a wrapping rope (connects the spring and cam) with respect to the cam-wheel increases while the moment arm between a Bowden cable (crossing the shoulder) and constant-radius wheel stays the same. Thus, the cam-wheel functions as a gearing mechanism that modulates the Bowden cable's pulling force according to the moment arm ratio between the cam and wheel.

The Bowden cable was clamped at the superior aspect of the shoulder using a self-aligning spherical bearing. The bearing allowed the Bowden cable to pivot and align with the arm across a large range of elevation plane angles. All components were mounted on a custom back brace, while the Bowden cable was attached to an arm cuff. provides mechanical assistance through a soft, cable-driven mechanism instead of rigid links, which (1) enables us to place forcegenerating components (i.e. the cam-wheel and spring) away from the shoulder joint, (2) permits shoulder rotations to occur freely about biological axes of rotation without conflicting with axes of rigid linkages, and (3) reduces the exoskeleton's weight. Second, the geometry of the cam-wheel gearing component can be customized to "tune" the gravity-compensating force to support a wide range of tasks and users. Finally, with our compact spring-cam-wheel module design, the exoskeleton can maintain a low profile.

\section{Calculation of Shoulder Elevation Moment due to Gravity}

24 The magnitude of the gravity moment about the shoulder depends on the upper extremity posture, while the orientation of the gravity moment depends on the orientation of the torso with respect to gravity. In our design calculations, we assumed that the 
negative shoulder elevation and, thus, can be calculated with a planar model of the arm. The shoulder gravitational moment was calculated as:

$$
\tau_{G}(\alpha, \beta)=W_{1} l_{1} \sin (\alpha)+W_{2}\left(\mathrm{~L} \sin (\alpha)+l_{2} \sin (\alpha+\beta)\right)
$$

where $W_{1}$ is the weight of the arm; $W_{2}$ is the sum of the forearm and hand weight; $l_{1}$ is the distance from the glenohumeral joint center to the arm's center of gravity; $l_{2}$ is the distance from the elbow joint center to the center of gravity of the combined forearmhand segment; $\mathrm{L}$ is the length of the arm segment; and $\alpha$ and $\beta$ are the shoulder elevation and elbow flexion angles, respectively (Fig. 1). Equation (1) shows that the gravitational moment depends on both shoulder and elbow posture and increases nonlinearly as the shoulder elevates from $0^{\circ}$ to $90^{\circ}$. We estimated the value of $\tau_{G}$ in (1) based on anthropometric data of a $50^{\text {th }}$ percentile male (age 27.45(5.64) years) and assuming a fully extended elbow [26, 27].

$$
\tau_{G}(\alpha, 0)=10.4 \sin (\alpha)(\mathrm{Nm})
$$

\section{Selection of Elastic Spring Component}

The force-generating components of previous passive exoskeletons are typically either zero-free-length springs, constant-force springs [28], or rubber bands [29]. For the shoulder joint, which has the largest range of motion of all human joints, an ideal passive element must be able to generate force over large deflections. We selected a constant-torque spring (Fig. 2) which has a compact design since their deflection is achieved by rotating a set of spools. The constant-toque spring also simplifies the design of the cam since it makes the shoulder gravity moment the only variable in equation (5). In a constant-torque spring, a coiled, elastic metal strip is wrapped over a storage spool. The metal strip is stretched by wrapping and rotating it around an output spool in the opposite direction in which it is coiled. Energy is released from the spring when the metal strip unwinds from the output spool back onto the storage spool. When the spring is stretched, it generates constant torque $\tau_{\text {spring }}$ about the axis of the output spool [30]. We converted the torque into a tension force in a wrapping rope by attaching a pulley to the output spool (Fig. 2).

Based on the gravity moment in (2) and equation (5), we selected a commercially available constant-torque spring that can nominally produce a torque, $\tau_{\text {spring }}=0.84 \pm 0.08 \mathrm{Nm}$ when wound 10 turns around the output spool, according to the manufacturer (SV12J192, Vulcan Spring \& Manufacturing, Telford, PA, USA). The spring material is 301 Stainless steel and the design parameters recommended by the manufacturer are shown in the table in Fig. 2. 


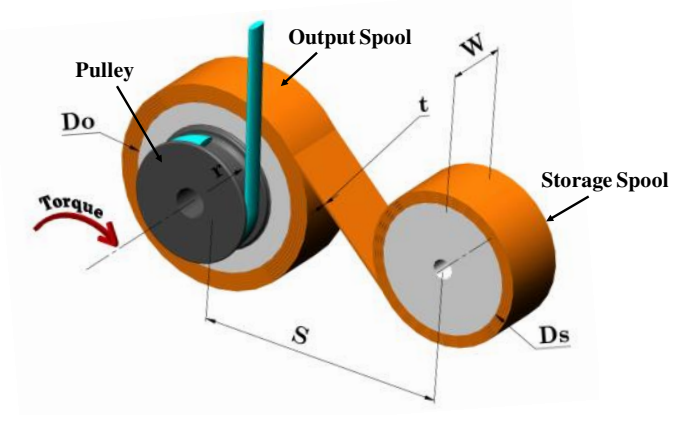

\begin{tabular}{|c|c|c|c|c|}
\hline $\boldsymbol{t}(\boldsymbol{m})$ & $\boldsymbol{W}(\boldsymbol{m})$ & $\boldsymbol{S}(\boldsymbol{m})$ & $\boldsymbol{D o}(\boldsymbol{m})$ & $\boldsymbol{D s}(\boldsymbol{m})$ \\
\hline 0.0003 & 0.016 & 0.66 & 0.062 & 0.036 \\
\hline
\end{tabular}

Fig. 2. Schematic view of a constant-torque spring. The geometrical properties of the selected spring assembly are listed in the table.

The torque generated by a constant-torque spring is higher during the stretching phase (i.e. spring winding onto the output spool) than during the recoil phase (i.e. spring winding back onto the storage spool). We opted to perform the design of WPCSE based on the torque generated during the recoiling phase during which it assists positive shoulder elevation movements. The torque, $0.02 \mathrm{Nm}$.

The cam-wheel is the key component of the WPCSE that adjusts the cable pulling force to output the desired increasing positive shoulder elevation moment with increasing shoulder elevation angle (Fig. 3).

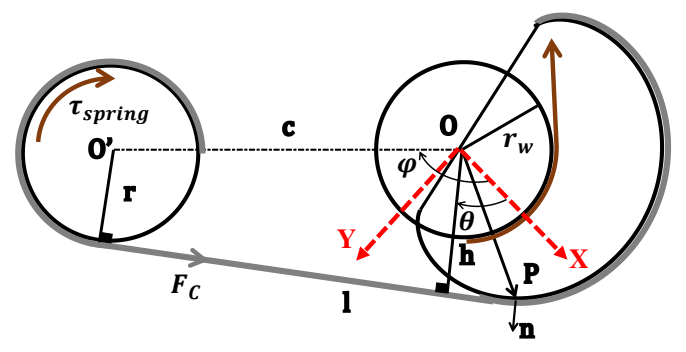

Fig. 3. Schematic representation of the spring-cam-wheel module. as (3): 


$$
\varphi=\frac{r_{s}}{r_{w}} \alpha
$$

where $r_{w}$ represents the radius of the constant-radius wheel and $r_{s}$ is the moment arm of the shoulder cable about the glenohumeral joint. Theoretically, the WPCSE can be designed to generate an assistive positive shoulder elevation moment $\tau_{E}$ that counteracts some proportion, $k$, of the gravity moment, $\tau_{G}(\alpha, 0)$. A value of $k=1$ is expected to completely balance the upper extremity weight in all static shoulder elevation postures. However, in this study, we set $k=1 / 4$, for three main reasons. First, during dynamic positive shoulder elevation movements, full static assistance coupled with the inertia of the moving upper extremity mass would exceed the negative shoulder elevation moment due to gravity. Second, our preliminary theoretical model did not account for friction and other factors that increase the WPCSE moment during negative shoulder elevation (Fig. 7), since the quantitative effect of these factors was unknown at the time of the study. Third, in a preliminary study of an exoskeleton prototype with $k=$ 1/2, some users needed to actively lower their arm with the WPCSE [31] to counteract the exoskeleton moment.

$$
\tau_{E}=k \tau_{G}(\alpha, 0)
$$

For the cam-wheel to be statically balanced, assuming no loss due to friction:

$$
F_{C}(\varphi) h(\varphi)-\frac{r_{w}}{r_{s}} \tau_{E}=0
$$

where $F_{C}(\varphi)$ is the tension force in the wrapping rope that connects the constant-torque spring to the variable-radius cam, and $h(\varphi)$ is the moment arm of the wrapping rope with respect to the cam's axis of rotation.

We first determined the form of $h(\varphi)$ in (5) to calculate the final geometry of the cam. The value of $r_{s}$ was set to $0.06 m$, which we estimated from a computational musculoskeletal model [32]. The cable tension force was calculated by dividing $\tau_{\text {spring }}$ by the radius $r$ of the pulley which is attached to the output spool. To determine the form of $h(\varphi)$ and values of $r_{w}$ and $r$, we performed a constrained, global numerical optimization using the GlobalSearch function in MATLAB (MathWorks, Inc., Natick, MA). The optimization identified parameter values that minimized the sum of the squared error between the actual $\left(\tau_{E}\right)$ and desired $\left(\frac{1}{4} \tau_{G}\right)$ assistive shoulder elevation moment by the exoskeleton, $\left\|\tau_{E}-\frac{1}{4} \tau_{G}\right\|^{2}$. From among five forms for $h(\varphi)$, including linear, quadrature, polynomial, power, and sinusoidal functions, that we tested in our global optimization procedure, we chose a sinusoidal form because it generated the lowest error [31]. This makes sense since the form of $\tau_{G}$ in (2) is also sinusoidal. 


$$
h(\varphi)=A \sin (B \varphi)+C
$$
coordinate of the contact point, $P$, between the wrapping rope and the cam surface (Fig. 3), can be represented as:

$$
\vec{P}=c e^{i \varphi}+e^{i \theta}(r-l i)
$$
where $c$ is the center-to-center distance between cam-wheel and the spring output spool; $l$ is the length of the unwrapped portion of the wrapping rope; and $\theta$ is the angle of the moment arm. The angle $\varphi$ can be written as a function of the moment arm $h(\varphi)$ :

$$
\varphi=\cos ^{-1}\left(\frac{h-r}{c}\right)+\theta
$$

9 We set $c=0.010 m$ to have the final integrated spring-cam-wheel module as compact as possible while still maintaining clearance between the cam-wheel component and the constant-torque spring. The value of $l$ was derived using the contact condition between

11 the cable and the cam $\vec{n} \cdot\left(\frac{\overrightarrow{d P}}{d \varnothing}\right)=0$. This leads to the following equation to calculate $l$.

$$
l=C \frac{d \varphi}{d \theta} \sin (\varphi-\theta)
$$

12 where $\frac{d \varphi}{d \theta}$ can be determined by taking the derivative of (8):

$$
\frac{d \varphi}{d \theta}=1-\frac{1}{\sqrt{C^{2}-(h-r)^{2}}} \frac{d h}{d \theta}
$$

13 Equations 7 to 10 are sufficient to develop the final profile of the cam-wheel (Fig. 4A). The coordinates of three hundred points 14 representing the cam profile were obtained to develop its computer-aided design (CAD) model (Fig. 4B). 


\section{Exoskeleton Prototype}

Based on the design parameters and dimensions above, we fabricated a prototype of the WPCSE using off-the-shelf and 3Dprinted components, as described below.

$4 \quad$ Spring-cam-wheel Module Assembly

The CAD models of the cam-wheel, spring spools, and pulley were developed in Solidworks software (2018, Dassault Systémes SolidWorks Corp., MA, USA). From the CAD models, the parts were 3D printed in ABS plastic (Fig. 4B, 4C, 4D). The constanttorque spring and output pulley were assembled and installed on a 304 -alloy stainless-steel sheet $(14 \mathrm{~cm} \times 17 \mathrm{~cm} \times 0.16 \mathrm{~cm})$ using Clevis pins and retainer rings. To facilitate the rotation of the spools, two deep-groove ball bearings $(688$-ZZ $8 \mathrm{~mm} \times 22 \mathrm{~mm} \times 7 \mathrm{~mm})$ were pressed in each spool. The cam-wheel was equipped with a single row ball bearing (Koyo, EE4C3) that was bolted to the stainless sheet. An inelastic, solid-braid nylon rope $(9.50 \mathrm{~mm}$ thickness) connected the cam and spring pulley. To prevent the constant-torque spring from suddenly winding onto the storage spool, a mechanical stopper (pin inside circular groove) was

A

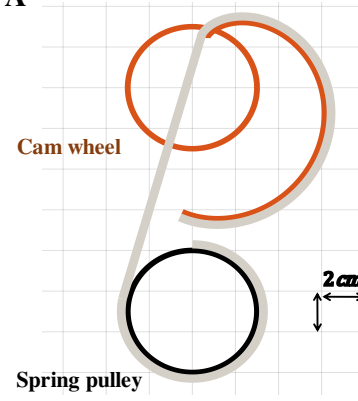

B

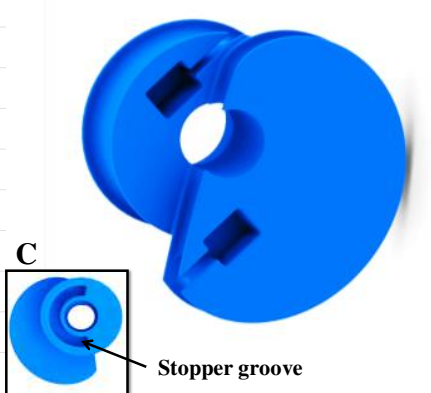

D

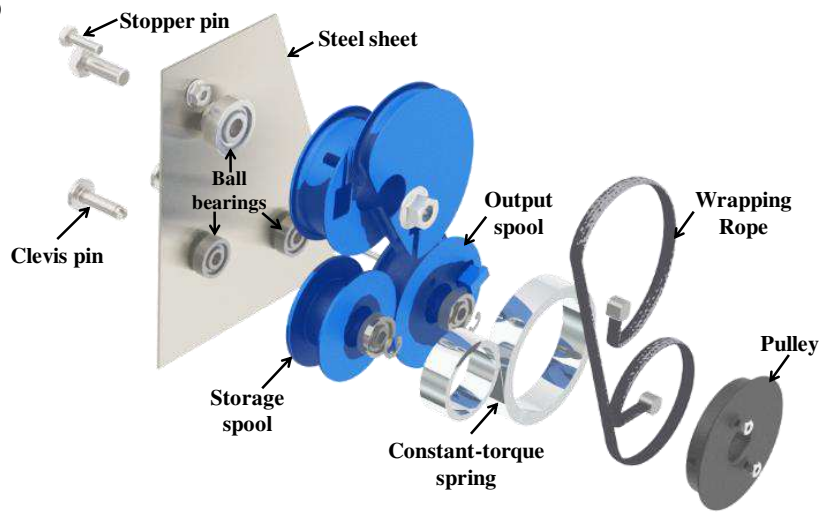

Fig. 4. A) Profile of the cam-wheel. B) CAD model of the cam-wheel. C) Posterior view of the cam-wheel. D) Exploded view of the spring-cam-wheel module assembly. 
incorporated in the output spool to limit its rotation to $350^{\circ}$. A similar stopper was incorporated in the cam-wheel to limit its rotation such that the WPCSE applied force to the arm for shoulder elevation angles between $0^{\circ}$ and $90^{\circ}$.

\section{Custom Braces}

The WPCSE prototype consisted of a back brace and an arm cuff, on which other components were mounted. We custom-made a thermoplastic back brace (acrylic sheet) by casting a person's torso with plaster gauze and fabricating positive and negative molds. The back brace was attached to a back-support vest that straps across the shoulder and around the waist (Fig. 5A). The inside of the back brace was covered with padding to improve fit and comfort. A thermoplastic cuff was attached to an adjustable, slide-on elbow support that straps to the arm both above and below the elbow joint (Fig. 5A). This prevented the arm cuff from sliding proximally while still permitting elbow flexion.

\section{Cable Routing}

A Bowden cable transmitted the force from the spring-cam-wheel module assembly to the arm cuff. A Bowden cable consists of an inner cable (commonly of steel) and an outer casing (a composite of helical steel wire and a plastic outer sheath). The inner wire wrapped around the wheel on one end and connected to the arm cuff on the other end using a spring snap.

\section{Prototype Specifications}

The Bowden cable casing was clamped at each end and in the middle by self-aligning spherical bearings (maximum pivot angle $=60^{\circ}$ ) that were bolted to the back brace (Fig. 5B). Clamping the casing was required so that the inner wire could move relative to it and transmit the pulling force. The spherical bearings permitted the cable to pivot and align with the arm as elevation plane angle changed. The path of the Bowden cable was designed to minimize friction (i.e. by avoiding bends with small radius of curvature). A small $(\sim 5 \mathrm{~cm})$ length of casing extended distally from the bearing mounted over the shoulder to enable the inner cable to curve smoothly over the shoulder.

1 The final prototype of the WPCSE weighed $1.82 \mathrm{~kg}$, of which $0.83 \mathrm{~kg}$ was due to the spring-cam-wheel module assembly, 0.82 $\mathrm{kg}$ was due to the back brace, and $0.17 \mathrm{~kg}$ was due to the arm cuff. The total weight of the WPCSE is relatively low and mostly borne on the torso, so we do not expect it to have a large effect on user fatigue. It was also designed to be donned/doffed independently by the user. However, the length of the inner wire of the Bowden cable needed to be adjusted once for each user according to their anthropometry. 


\section{Benchtop Setup}

In addition to the WPCSE prototype, we developed a benchtop prototype (Fig. 5C) to preliminarily evaluate the mechanical output of the WPCSE and its components. The benchtop prototype consisted of a v-belt pulley (radius $=0.08 \mathrm{~m}$ ) representing the Bowden cable wrapping over the shoulder; the pulley had an embedded handhold so that it could be rotated manually to mimic shoulder elevation movements. Depending on the test (Experiments section), the v-belt pulley was connected to either the springcam-wheel assembly or to the WPCSE. A rotary encoder (LPD-3806-600bm-G5-24c, GTEACH, China) was installed on top of the v-belt pulley to measure its rotation angle for computing the mimicked shoulder elevation angle. An s-type load cell (ATOLC-S04, 50kg capacity, ATO, Diamond Bar, CA, USA) was also placed along the cable path to measure its pulling force. An Arduino Nano board was programmed to compute the shoulder elevation moment and angle from the measured force and angle, respectively.

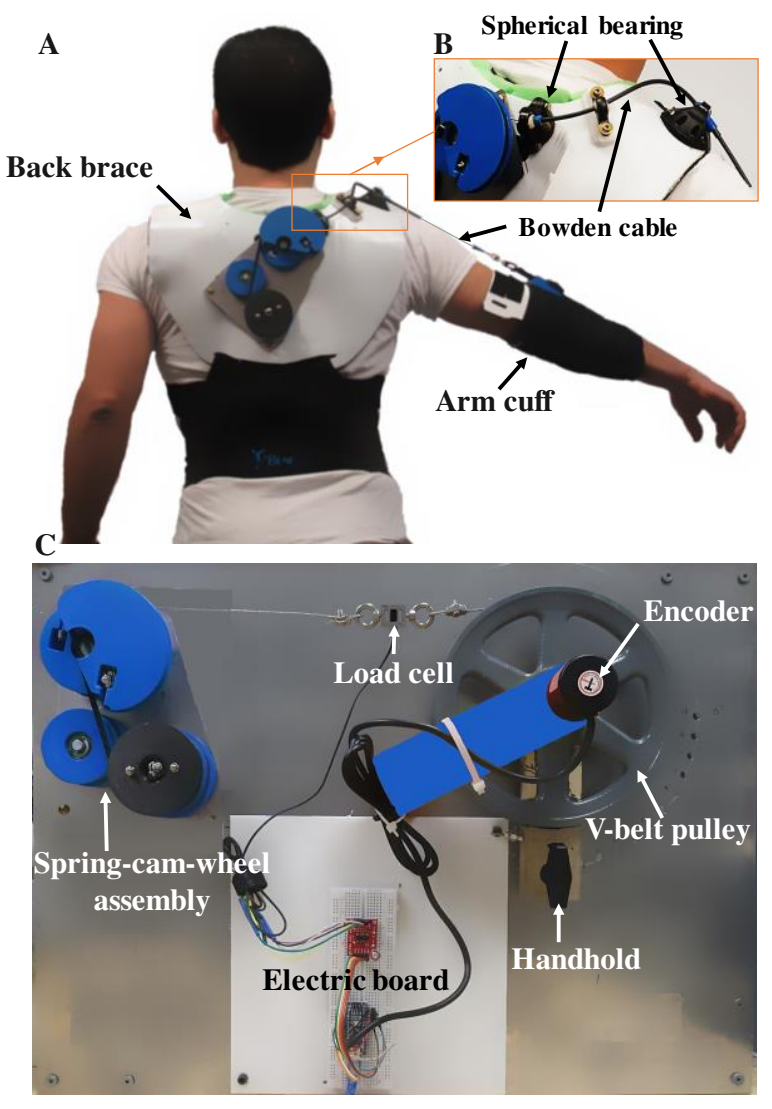

Fig. 5. A) WPCSE prototype. B) Bowden cable routing path over the shoulder. C) Benchtop prototype. 


\section{Experiments}

\section{Mechanical Performance Evaluation}

Using the benchtop prototype, we measured the torque generated by the spring alone, the spring-cam-wheel module alone, and the entire WPCSE. The spring-cam-wheel module and WPCSE were essentially the same except that the WPCSE also included the Bowden cable and its routing path on the back brace. The WPCSE was placed on the fabricated positive mold of the torso and fixed to a workbench. The side of the Bowden cable that attaches to the arm cuff was connected to the v-belt pulley. To test the spring-cam-wheel module alone, we fixed it to the benchtop board and connected the wheel to the v-belt pulley via the inner Bowden cable (Fig. 5C). The v-belt pulley was manually rotated from $0^{\circ}$ to $67.5^{\circ}$ to simulate the shoulder elevation from $0^{\circ}$ to $90^{\circ}$. The angle difference was due to the difference between the v-belt pulley radius and the estimated moment arm of the Bowden cable about the shoulder.

The v-belt pulley was rotated manually counterclockwise (i.e. simulated positive shoulder elevation) and clockwise (i.e. simulated negative shoulder elevation), three times at a slow speed (i.e. quasi-static condition). Cable pulling force and pulley rotational angle data were collected at $20 \mathrm{~Hz}$. The shoulder elevation moment was calculated by multiplying the cable pulling force

by the radius of the v-belt pulley. Measured pulley rotation angles were multiplied by $\frac{4}{3}$ (pulley-to-shoulder moment arm ratio) to compute the simulated shoulder elevation angles. The final moment-angle relationships for the spring-cam-wheel module and WPCSE were obtained by resampling the simulated positive and negative shoulder elevation phases into 50 points each and averaging across the three repetitions.

To test the constant-torque spring alone (i.e. pre-cam), the spring was installed on the benchtop board. The output pulley was attached to the v-belt pulley via an inner Bowden cable. We wound the spring strip 10 turns around the output spool. The torque of the spring was calculated by multiplying the cable tension force by the radius of output pulley $(0.03 m)$. We computed the spring's torque-angle relationship as described above for the spring-cam-wheel module and WPCSE.

\section{Biomechanical Performance Evaluation}

1) Participants

We conducted a pilot study to preliminarily determine the effect of the WPCSE on the neuromuscular activity of muscles crossing the shoulder and on shoulder kinematics. We recruited four able-bodied participants ( 3 males and 1 female, age $=$ 
The experimental procedure was approved by the institutional review board at the University of Tennessee (UTK IRB-19-04990three shoulder movements with and without assistance (Fig. 6A). For the without-assistance condition, the Bowden cable was
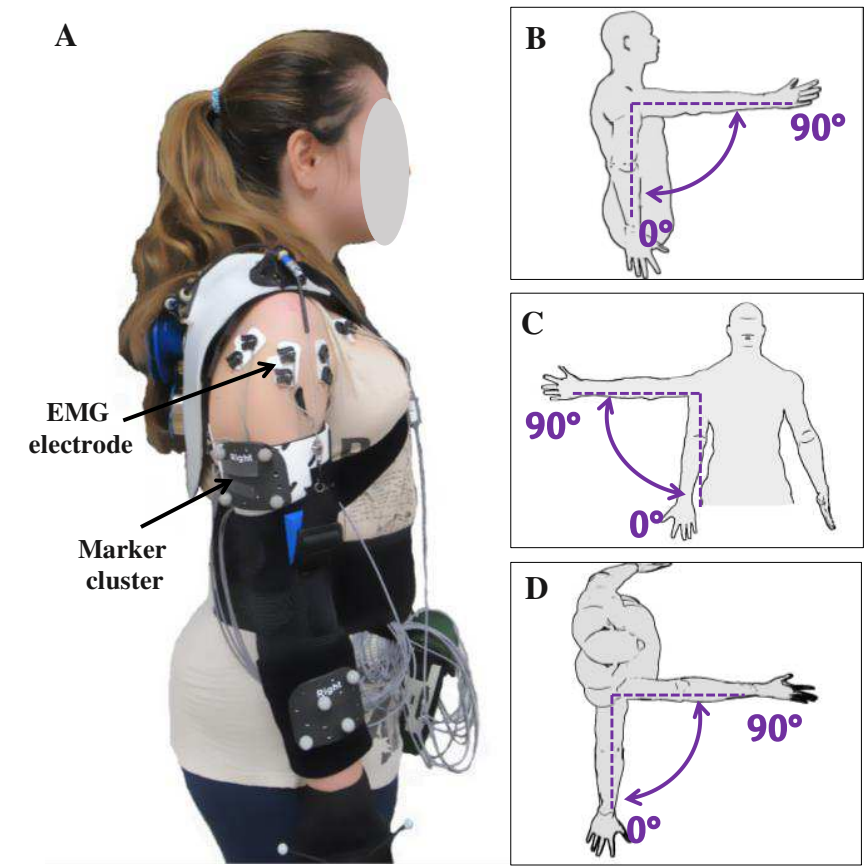

Fig. 6. A) A participant with WPCSE, EMG electrodes, and reflective marker clusters. B). Shoulder elevation in the sagittal plane. C) Shoulder elevation in the frontal plane. D) Shoulder adduction/abduction in the horizontal plane.

detached from the arm cuff. The movements were shoulder elevation in the sagittal and frontal planes (Fig. 6B, 6C), and shoulder adduction/abduction in the horizontal plane (Fig. 6D). For each trial, participants moved the shoulder from $0^{\circ}$ to $90^{\circ}$ and back to $0^{\circ}$ in the relevant plane (Fig. 6). The trials were performed with the elbow fully extended, at the participant's preferred speed, and with a brief (1-2 seconds) pause at the upper $\left(90^{\circ}\right)$ and lower $\left(0^{\circ}\right)$ limit of movement. In each trial, participants performed 10 continuous repetitions of each shoulder movement while sitting upright on a chair. We randomized the order of exoskeleton condition (with and without WPCSE) and, within each exoskeleton condition, randomized the order of shoulder movements. Participants rested between trials for about 30 seconds to reduce the likelihood of fatigue. 
During the trials, we synchronously measured electromyograms (EMG) and kinematics of the participants. We recorded EMG from several muscles crossing the shoulder, including anterior (AD), middle (MD), and posterior deltoid (PD), pectoralis major (PM), latissimus dorsi (LD), infraspinatus (ISP), trapezius (TRAP), biceps brachii (BB), and triceps brachii (TB) (Fig. 6A). EMG data were recorded at a sampling frequency of $3000 \mathrm{~Hz}$ (TeleMyo $2400 \mathrm{G} 2$, Noraxon, AZ, USA) using surface electrodes (bipolar silver/silver chloride electrodes) attached to the participant's skin. Before starting the experiment trials, baseline EMG of muscles at rest and during maximum voluntary contraction (MVC) were recorded based on a previously described method [34]. We used a 7-camera infrared motion capture system (OptiTrack Prime 13) to track the three-dimensional positions of reflective marker clusters that were placed on the participant's upper back, arm, forearm, and hand [35]. Marker cluster position data were recorded 9 at $120 \mathrm{~Hz}$.

\section{3) Data Processing}

The EMG data for each movement repetition were divided into positive elevation/adduction, and negative elevation/abduction phases based on the kinematics. The raw EMG data was band-pass filtered at 10-500 Hz, full-wave rectified, and low pass filtered with a $4^{\text {th }}$ order Butterworth filter at a cut-off frequency of $6 \mathrm{~Hz}$. For each participant, the processed EMG for each muscle were normalized by the maximum processed-EMG value recorded from the respective muscle during MVCs. calculated and averaged across eight middle repetitions (i.e. out of 10 repetitions) for each trial and movement phase [36, 37].

The MotionMonitor software (V8.0, Innovative Sports Training, Inc., Chicago, IL, USA) was used to compute and resample (at $3000 \mathrm{~Hz}$ ) the shoulder elevation and plane of elevation angles from the marker cluster position data. Shoulder elevation angle (sagittal and frontal trials only) or elevation plane angle (horizontal trials only) was considered for further analyses since movements were predominantly along these degrees of freedom. Angular velocity was also calculated by taking the derivative of the predominant shoulder angles.

\section{Results}

\section{Mechanical Performance}

Under quasi-static conditions, the constant-torque spring was able to produce a nearly constant torque $(0.77 \pm 0.02 \mathrm{Nm})$ that was in the range of the manufacturer's specified nominal torque $(0.84 \pm 0.08 \mathrm{Nm})$ for the stretching phase (Fig. 7$)$. The torque 
For the spring-cam-wheel module assembly, the measured moment matched reasonably well with the theoretical moment during simulated positive shoulder elevation. This was expected because we optimized the design parameters of the cam-wheel based on the torque value that spring generates during its unloading phase. However, a larger moment, up to 50\% larger than the theoretical

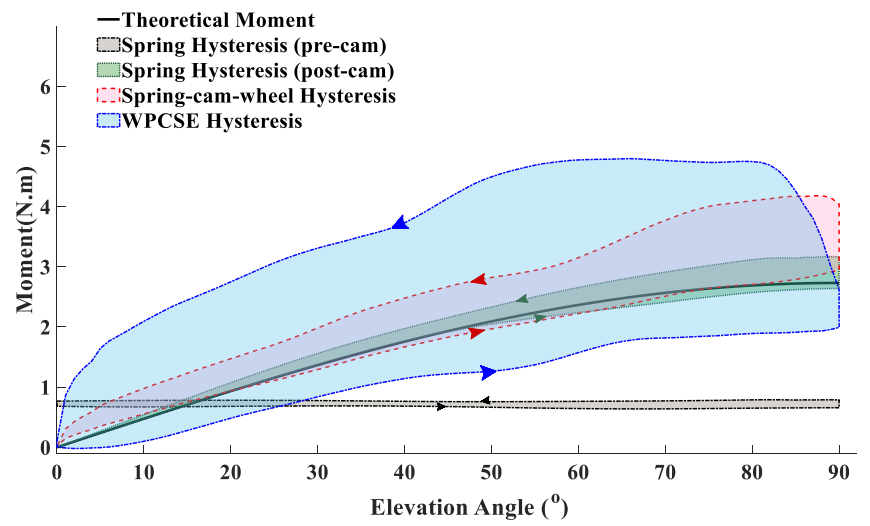

Fig. 7. Joint moment produced by the constant-torque spring (pre- and postcam), spring-cam-wheel module, and WPCSE during simulated shoulder elevation. The shaded regions indicate the degree of hysteresis, which is the difference in torque or moment between the positive and negative shoulder elevation phases.

moment, was required during simulated negative shoulder elevation to stretch the spring. The difference in moment between simulated positive and negative shoulder elevation phases was even greater for the WPCSE (Fig. 7, blue area); the measured WPCSE moment was up to $30 \%$ lower than the theoretical moment during simulated positive shoulder elevation, and up to $120 \%$ higher than the theoretical moment during simulated negative shoulder elevation.

\section{Biomechanical Performance}

During positive elevation/adduction, nRMS EMG values of several muscles tended to be lower for trials with exoskeleton assistance across the participants (Fig. 8). The reduction in muscle activity occurred across all four subjects for the AD (22-47\%), MD (19-50\%), PD (16-38\%), ISP (20-40\%), TRAP (5-48\%), and LD (27-55\%) muscles during positive shoulder elevation in the sagittal plane; for the AD (12-27\%), MD (18-36\%), ISP (14-50\%), and TRAP (27-39\%) muscles during positive shoulder elevation in the frontal plane; and for the MD (4-32\%), PD (13-36\%), ISP (12-34\%), TRAP (12-43\%), and LD (2-31\%) muscles during horizontal adduction. During negative shoulder elevation/abduction with exoskeleton assistance (Fig. 9), nRMS EMG was lower for all subjects for the ISP (4-35\%) and TRAP (18-50\%) muscles in sagittal plane; for the AD (3-40\%), MD (7-37\%), ISP (8-50\%), and TRAP (22-30\%) muscles in frontal plane; and for the MD (9-21\%), PD (10-25\%), ISP (4-34\%), and TRAP (18-43\%) muscles in the horizontal plane. 
Similarly, during the positive shoulder elevation/adduction phase, the nPeak EMG values of several muscles trended lower with exoskeleton assistance than without (Fig. 11, supplementary); the difference occurred across all four subjects for the AD (5-35\%), MD (15-30\%), LD (20-55\%), and ISP (23-27\%) muscles for positive elevation in the sagittal plane; AD (6-23\%), MD (2-31\%), and TRAP (17-30\%) muscles for positive elevation in the frontal plane; and MD (6-32\%), PD (11-33\%), LD (3-33\%), ISP (9-

\section{Positive Elevation/Abduction}

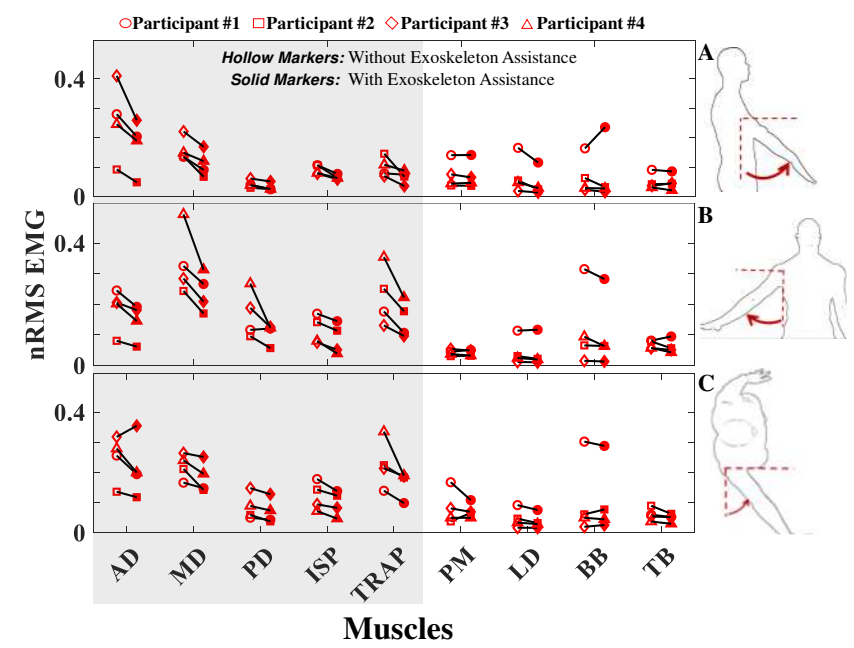

Fig. 8. Root mean square of the normalized EMG (nRMS EMG) of several muscles crossing the shoulder during positive shoulder elevation in the sagittal plane (A), shoulder elevation in the frontal plane (B), and horizontal adduction (C) with and without exoskeleton assistance. Each marker shows the mean nRMS EMG across movement repetitions for each subject. Hollow and solid markers indicate trials without and with exoskeleton assistance, respectively. Shaded area shows muscles that primarily contribute force in the direction of shoulder elevation, based on their moment arms.

35\%), and TRAP (11-41\%) muscles for horizontal adduction movements. During the negative elevation/abduction phase (Fig. 12, supplementary), the values of nPeak EMG were reduced across all subjects with exoskeleton assistance for the TRAP (11-20\%) muscle during shoulder elevation in the frontal plane; and for the MP (9-50\%), ISP (15-41\%), and TRAP (5-41\%) muscles during horizontal abduction movements. In some cases, muscle activity was higher with exoskeleton assistance than without, but not consistently across all four subjects for any muscle and movement.

Qualitatively, during the three tested movements that subjects performed at a self-selected speed, each subject's shoulder kinematics changed with assistance in different ways (Fig. 10). For example, during the shoulder elevation movements with exoskeleton assistance, participant 1 had lower and higher shoulder elevation angles during the positive and negative elevation portions of the movement, respectively. For the same movements, the shoulder elevation angles of participant 3 were relatively

14 similar with and without exoskeleton assistance. Although the movement durations were longer (0.1s to $0.4 \mathrm{~s})$ for trials with 
exoskeleton assistance, the subjects attained the upper limit (i.e. $90^{\circ}$ ) at approximately $50 \%$ of the movement duration both with and without assistance. Participants noted subjectively that the WPCSE did not constrain their motion during the tested movements.

The normalized angular velocity for all but participant 1 was lower with assistance than without, especially during shoulder elevation movements in the sagittal and frontal planes (Fig. 13, supplementary). However, qualitatively, the angular velocity profiles (i.e. the overall shape of the curves) and the durations of the acceleration/deceleration phases are similar between assistance conditions.

\section{Discussion}

We presented the design of a wearable passive cam-based shoulder exoskeleton (WPCSE), whose novel feature is a spring-camwheel module that can provide nonlinear, customizable mechanical output; in our case, the mechanical output assists the shoulder in the direction of positive shoulder elevation based on the configuration of a force transmission cable. The customized assistance

11 is made possible specifically by the variable-radius cam, which acts as a gearing mechanism to modulate the force output of the 12 spring. Cams are excellent design features that modulate force according to their shape. Thus, cams have been used in many other 13 passive mechanisms, including gravity-compensating passive shoulder exoskeletons [38]. Customizable assistance is an essential 14 feature of exoskeletons since assistance requirements vary (1) across users as a function of their anthropometry and functional

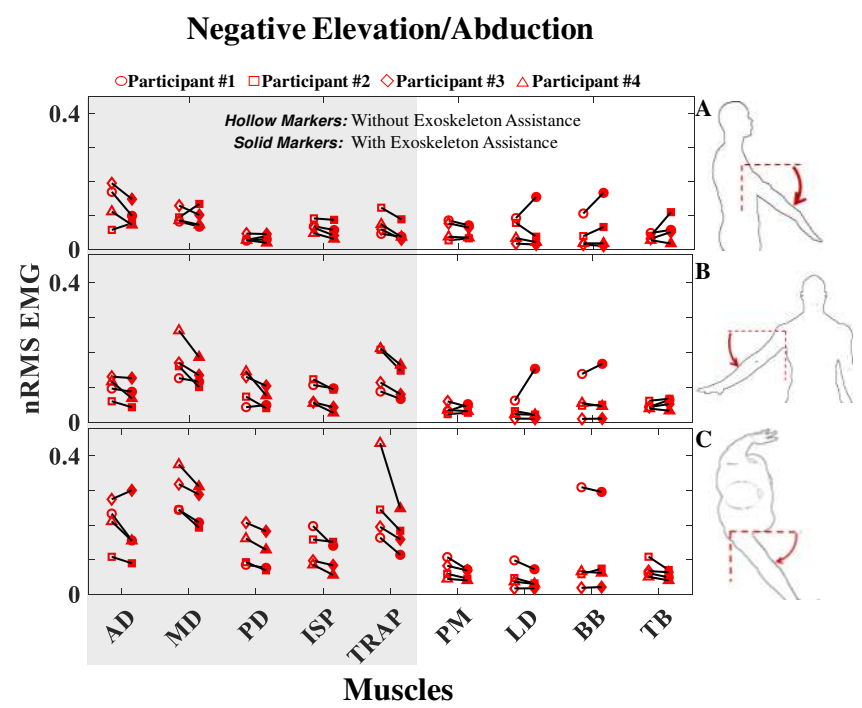

Fig. 9. Root mean square of the normalized EMG (nRMS EMG) of several muscles crossing the shoulder during negative shoulder elevation in the sagittal plane (A), negative shoulder elevation in the frontal plane (B), and horizontal abduction (C) with and without exoskeleton assistance. Each marker shows the mean nRMS EMG across movement repetitions for each subject. Hollow and solid markers indicate trials without and with exoskeleton assistance, respectively. Shaded area shows muscles that primarily contribute force in the direction of shoulder elevation, based on their moment arms. 
ability level and (2) across applications and tasks.

For both the spring-cam-wheel module and the WPCSE, the moment measured during the mechanical performance evaluation was larger during negative shoulder elevation (i.e. as the spring was being stretched) than during positive shoulder elevation (i.e. as the spring recoiled). We suspect that this so-called hysteresis behavior was primarily due to friction, for instance, at bearings and between the cables and components they wrap around. Inefficiency in the energy storage and return within the spring itself, inherent in all elastic springs, is another potential source of loss. The quantitative friction data from this study will be incorporated in our theoretical model so that it can be accounted for in future design iterations of our WPCSE prototype.

The hysteresis effect was more dramatic (i.e. more than 2x larger) with the WPCSE, which included the Bowden cable, than with the spring-cam-wheel module alone. Bowden cables are advantageous in wearable robotics applications in terms of design flexibility and remote actuation. However, Bowden cables introduce considerable friction [39] and appeared to be the main cause for the hysteresis behavior in the WPCSE. This friction, which originates from the contact between the inner wire and outer casing of the Bowden cable, is considered to be a nonlinear function of multiple geometric and material properties [40]. In the design of the WPCSE, we tried to minimize the Bowden cable friction by (1) fixing the cable's routing path using self-aligning spherical bearings and (2) placing the bearings so that the cable followed the most direct and shortest path from the spring-cam-wheel module to the shoulder and arm cuff. For future WPCSE design iterations, we will explore other Bowden cable configurations and alternative force transmission methods to reduce friction.

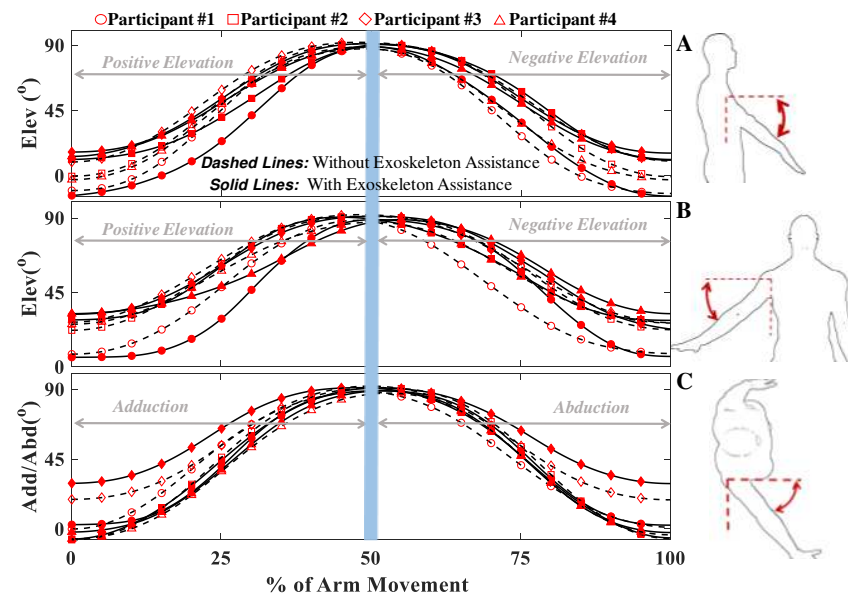

Fig. 10. Shoulder angular displacement during elevation in the sagittal plane (A), elevation in the frontal plane (B), and horizontal adduction/abduction (C) with and without exoskeleton assistance. Each curve shows the mean of dominant angular displacement across movement repetitions for each subject. Hollow markers with dashed lines indicate trials without exoskeleton assistance, and solid markers with solid lines indicate trials with the exoskeleton assistance. Blue bar shows the approximate location of the upper limit of shoulder movements. 
Promisingly, while only compensating for one-fourth of the gravity moment, the WPCSE showed a trend of reducing the root mean square (ranging from $2 \%$ to $50 \%$ ) and peak (ranging from $5 \%$ to $55 \%$ ) normalized EMG of several muscles crossing the shoulder consistently across all four subjects. Reductions in muscle activity were mostly consistent across the subjects for the deltoid and rotator cuff muscles, which play major roles in compensating for gravity and stabilizing the glenohumeral joint during shoulder movements [41]. Our results are consistent with several previous studies that also showed that varying levels of gravity compensation at the shoulder can reduce muscle activity $[8,9,42,43]$. The results of our pilot study justify and facilitate the design of a biomechanical study with greater statistical power (i.e. larger sample size) to validate the efficacy of the WPCSE. To support clinical translation, it will also be important to identify what magnitude of muscle activity reductions are clinically meaningful; this likely depends on factors such as pathology (e.g. rotator cuff tear vs nerve injury) and treatment objective (e.g. improve motor function vs offload tissues).

Interestingly, during negative elevation/abduction movements, the WPCSE tended to reduce the activity of the anterior deltoid, posterior deltoid, trapezius, and/or infraspinatus muscles across all four subjects. This indicates that the participants were likely able to lower their arm using mostly gravity, even though the moment generated by the WPCSE during negative shoulder elevation was considerably higher than predicted by our theoretical model. This result, promisingly, contrasts with our previous computational study, which predicted higher activity in several muscles during negative shoulder elevation [32]. One possible explanation is that, during such movements, the WPCSE offloads muscles that contribute to positive shoulder elevation and are usually eccentrically contracted to control the shoulder's angular descent. Additionally, participants may adapt their kinematics in subtle ways to avoid increasing muscle activity. We will test these hypotheses in a future, more comprehensive biomechanical study of the WPCSE. continuously move their arms between them, suggesting that the WPCSE did not constrain subjects' motions during the tested movements; this was supported by verbal feedback from the subjects that the WPCSE did not constrain their motions. The shape of angular velocity profile was similar between with and without exoskeleton conditions. However, subjects chose to move slower with exoskeleton assistance, as indicated by longer movement durations and lower angular velocities with exoskeleton assistance than without. The slower movements may be due to subjects having no prior experience with the exoskeleton; in future studies, 
The level of mechanical assistance generated by the WPCSE is expected to strongly influence neuromuscular activity, joint loads, and motor function at the shoulder. A desirable level of assistance would be one that both maximally assists and minimally resists user-generated moments. For example, though a high level of gravity compensation at the shoulder would maximally assist positive shoulder elevation movements, it might also require the user to generate higher negative shoulder elevation moments to overcome the gravity compensation and lower the arm. More research is needed to investigate the relationship between exoskeleton assistance level and biomechanical performance, which will inform the future design and prescription of wearable passive shoulder exoskeletons.

We chose to compare biomechanics with and without exoskeleton assistance, rather than with and without the exoskeleton, for two main reasons. First, the former comparison controlled for any effect that simply wearing the exoskeleton components (independent of the assistance force) may have on biomechanics. Second, chosen comparison permitted a more rigorous study design; since subjects wore the exoskeleton for both assistance conditions, we could more easily and reliably randomize the order of trials of each condition while reducing within-condition variability (e.g. variation in exoskeleton placement due to repeated don/doff of the device). Since the without-assistance condition does not truly represent the subject's normal "free" movement, it will be important to include a without-exoskeleton condition in future biomechanics studies of the WPCSE.

This study has several limitations that should be addressed in future prototypes and studies. First, the mechanical testing was not comprehensive and did not include identification of mechanical properties of all individual components of the exoskeleton efficacy of the WPCSE to support clinical translation.

\section{Conclusion}


To address an unmet clinical need for movement assistance at the shoulder, we presented the design of a WPCSE based on a novel spring-cam-wheel module. As a first step toward clinical translation, we preliminarily evaluated the mechanical and biomechanical performance of our WPCSE prototype. Our results showed that the WPCSE, compensating for a modest one-fourth of the gravity moment at the shoulder, tended to reduce the activity of several muscles crossing the shoulder, including the deltoid and rotator cuff muscles. However, our mechanical evaluation revealed aspects of the design that limit the assistance that the WPCSE could provide. In our future work, we will test different WPCSE assistance levels and identify a range of assistance that most enhances shoulder motor function and biomechanics without impeding movements. More comprehensive biomechanical studies will be performed to assess the WPCSE for more biomechanical parameters (e.g. range of motion and endpoint/hand kinematics), more participants (both able-bodied subjects and people with shoulder disability), and more movements that typify activities of daily living. Finally, several design refinements, especially those that reduce friction in the system, will be explored.

\section{Declarations}

\section{Abbreviations}

13 WPCSE: Wearable Passive Cam-based Shoulder Exoskeleton. CAD: Computer-Aided Design. EMG: Electromyography. AD: 14 Anterior Deltoid. MD: Middle Deltoid. PD: Posterior Deltoid. PM: Pectoralis Major. LD: Latissimus Dorsi. ISP: Infraspinatus. 15 TRAP: Trapezius. BB: Biceps Brachii. TB: Triceps Brachii. MVC: Maximum Voluntary Contraction.

\section{Ethics approval and consent to participate}

17 This study was approved by the institutional review board at the University of Tennessee (UTK IRB-19-04990-FB, 2020), and all 18 participants provided their informed consent.

\section{Consent for publication}

20 Not applicable.

\section{Availability of data and materials}

22 Not applicable.

23 Competing interests

24 None.

$25 \quad$ Funding 
This study was supported by start-up funds from the Department of Mechanical, Aerospace and Biomedical Engineering at the

University of Tennessee. The authors thank Vulcan Spring \& Manufacturing for providing the constant-torque springs.

\section{Authors' contributions} manuscripts.

\section{Acknowledgements}

Not applicable.

\section{References}

1. $\quad$ Lawrence, E.S., et al., Estimates of the prevalence of acute stroke impairments and disability in a multiethnic population. Stroke, 2001. 32(6): p. 12791284.

2. Meislin, R.J., J.W. Sperling, and T.P. Stitik, Persistent shoulder pain: epidemiology, pathophysiology, and diagnosis. American journal of orthopedics (Belle Mead, NJ), 2005. 34(12 Suppl): p. 5-9.

3. Gerber, C., et al., Mechanical strength of repairs of the rotator cuff. The Journal of bone and joint surgery. British volume, 1994. 76(3): p. 371-380.

4. $\quad$ Crouch, D.L., et al., Relationship between maximum isometric joint moment and functional task performance in patients with brachial plexus injury: A pilot study. Gait \& posture, 2016. 44: p. 238-244.

5. Kolb, S.J. and J.T. Kissel, Spinal Muscular Atrophy. Neurologic clinics, 2015. 33(4): p. 831-846.

6. Sukal, T.M., M.D. Ellis, and J.P. Dewald, Shoulder abduction-induced reductions in reaching work area following hemiparetic stroke: neuroscientific implications. Experimental brain research, 2007. 183(2): p. 215-223.

7. Rosen, J., et al. The human arm kinematics and dynamics during daily activities - toward a 7 DOF upper limb powered exoskeleton. in ICAR '05. Proceedings., 12th International Conference on Advanced Robotics, 2005. 2005.

8. Coscia, M., et al., The effect of arm weight support on upper limb muscle synergies during reaching movements. Journal of NeuroEngineering and Rehabilitation, 2014. 11(1): p. 22.

9. Hall, P.T. and D.L. Crouch, Effect of continuous, mechanically passive, anti-gravity assistance on kinematics and muscle activity during dynamic shoulder elevation. Journal of Biomechanics, 2020: p. 109685.

10. Perry, J.C., J. Rosen, and S. Burns, Upper-limb powered exoskeleton design. IEEE/ASME transactions on mechatronics, 2007. 12(4): p. $408-417$.

11. Nef, T., M. Guidali, and R. Riener, ARMin III-arm therapy exoskeleton with an ergonomic shoulder actuation. Applied Bionics and Biomechanics, 2009. 6(2): p. 127-142.

12. Roderick, S., M. Liszka, and C. Carignan. Design of an arm exoskeleton with scapula motion for shoulder rehabilitation. in ICAR'05. Proceedings., 12th International Conference on Advanced Robotics, 2005. 2005. IEEE.

13. Frisoli, A., et al. A new force-feedback arm exoskeleton for haptic interaction in virtual environments. in First Joint Eurohaptics Conference and Symposium on Haptic Interfaces for Virtual Environment and Teleoperator Systems. World Haptics Conference. 2005.

14. Park, H., R. Yupeng, and Z. Li-Qun. IntelliArm: An exoskeleton for diagnosis and treatment of patients with neurological impairments. in 2008 2nd IEEE RAS \& EMBS International Conference on Biomedical Robotics and Biomechatronics. 2008.

15. Mao, Y., et al., Human movement training with a cable driven arm exoskeleton (CAREX). IEEE Transactions on Neural Systems and Rehabilitation Engineering, 2014. 23(1): p. 84-92.

16. Li, Z., et al. Design and control of a 4-DOF cable-driven arm rehabilitation robot (CARR-4). in 2017 IEEE International Conference on Cybernetics and Intelligent Systems (CIS) and IEEE Conference on Robotics, Automation and Mechatronics (RAM). 2017.

17. Rahman, T., et al., Passive exoskeletons for assisting limb movement. Journal of rehabilitation research and development, 2006. 43(5): p. 583.

18. Lucieer, P. and J.L. Herder. Design of an adjustable compensation mechanism for use in a passive arm support. in ASME 2005 International Design Engineering Technical Conferences and Computers and Information in Engineering Conference. 2005. American Society of Mechanical Engineers Digital Collection.

19. Sanchez, R., et al. Monitoring functional arm movement for home-based therapy after stroke. in The 26th Annual International Conference of the IEEE Engineering in Medicine and Biology Society. 2004.

20. de Vries, A., et al., The amount of support provided by a passive arm support exoskeleton in a range of elevated arm postures. IISE Transactions on Occupational Ergonomics and Human Factors, 2019: p. 1-11.

21. Nussbaum, M.A., et al., An Introduction to the Special Issue on Occupational Exoskeletons. IISE Transactions on Occupational Ergonomics and Human Factors, 2019. 7(3-4): p. 153-162.

22. Yasuda, Y.L., K. Bowman, and J.D. Hsu, Mobile arm supports: criteria for successful use in muscle disease patients. Archives of physical medicine and rehabilitation, 1986. 67(4): p. 253-256.

Atkins, M.S., et al., Mobile arm supports: evidence-based benefits and criteria for use. The journal of spinal cord medicine, 2008. 31(4): p. 388-393.

24. James, W. and J. Orr, Upper limb weakness in children with Duchenne muscular dystrophy-a neglected problem. Prosthetics and orthotics international, 1984. 8(2): p. 111-113 
25. Sanchez, R.J., et al., Automating Arm Movement Training Following Severe Stroke: Functional Exercises With Quantitative Feedback in a GravityReduced Environment. IEEE Transactions on Neural Systems and Rehabilitation Engineering, 2006. 14(3): p. 378-389.

26. McConville, J.T., Anthropometric relationships of body and body segment moments of inertia. Vol. 32. 1981: Air Force Aerospace Medical Research Laboratory, Aerospace Medical Division ....

27. Winter, D.A., Biomechanics and motor control of human movement. 2009: John Wiley \& Sons.

28. Hill, P.W., E.T. Wolbrecht, and J.C. Perry. Gravity Compensation of an Exoskeleton Joint Using Constant-Force Springs. in 2019 IEEE 16th International Conference on Rehabilitation Robotics (ICORR). 2019. IEEE.

29. Rahman, T., et al., Design and testing of a functional arm orthosis in patients with neuromuscular diseases. IEEE Transactions on Neural Systems and Rehabilitation Engineering, 2007. 15(2): p. 244-251.

30. McGuire, J.R. and J.A. Yura, Advances in the analysis and design of constant-torque springs. 1996.

31. Asgari, M., et al. Wearable Shoulder Exoskeleton with Spring-Cam Mechanism for Customizable, Nonlinear Gravity Compensation in 202042 th Annual International Conference of the IEEE Engineering in Medicine and Biology Society (EMBC). 2020. Montreal: Accepted.

32. Nelson, A.J., et al., Effect of Mechanically Passive, Wearable Shoulder Exoskeletons on Muscle Output During Dynamic Upper Extremity Movements: A Computational Simulation Study. Journal of Applied Biomechanics, 2020. 36(2): p. 59-67.

33. Tidwell, P.H., et al., Synthesis of wrapping cams. 1994.

34. Hodder, J.N. and P.J. Keir, Obtaining maximum muscle excitation for normalizing shoulder electromyography in dynamic contractions. Journal of Electromyography and Kinesiology, 2013. 23(5): p. 1166-1173.

35. Wu, G., et al., ISB recommendation on definitions of joint coordinate systems of various joints for the reporting of human joint motion-Part II: shoulder, elbow, wrist and hand. Journal of biomechanics, 2005. 38(5): p. 981-992.

36. Merletti, R. and P. Di Torino, Standards for reporting EMG data. J Electromyogr Kinesiol, 1999. 9(1): p. 3-4.

37. Kim, S., et al., Assessing the influence of a passive, upper extremity exoskeletal vest for tasks requiring arm elevation: Part I- "Expected" effects on discomfort, shoulder muscle activity, and work task performance. Applied ergonomics, 2018. 70: p. 315-322.

38. Schroeder, J.S. and J.C. Perry. Development of a series wrapping cam mechanism for energy transfer in wearable arm support applications. in 2017 International Conference on Rehabilitation Robotics (ICORR). 2017. IEEE.

39. Chen, D., Y. Yun, and A.D. Deshpande. Experimental characterization of bowden cable friction. in 2014 IEEE International Conference on Robotics and Automation (ICRA). 2014. IEEE.

40. Schiele, A., et al. Bowden cable actuator for force-feedback exoskeletons. in 2006 IEEE/RSJ International Conference on Intelligent Robots and Systems. 2006. IEEE.

41. Halder, A.M., E. Itoi, and K.-N. An, Anatomy and biomechanics of the shoulder. Orthopedic Clinics, 2000. 31(2): p. 159-176.

42. Prange, G.B., et al., Influence of gravity compensation on muscle activation patterns during different temporal phases of arm movements of stroke patients. Neurorehabilitation and neural repair, 2009. 23(5): p. 478-485.

43. Prange, G.B., et al., Influence of gravity compensation on muscle activity during reach and retrieval in healthy elderly. Journal of electromyography and kinesiology, 2009. 19(2): p. e40-e49. 


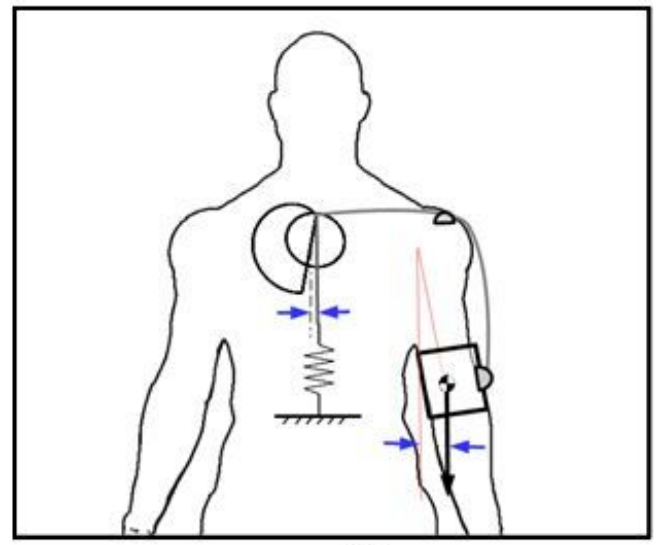

$\alpha \approx 0^{\circ}$

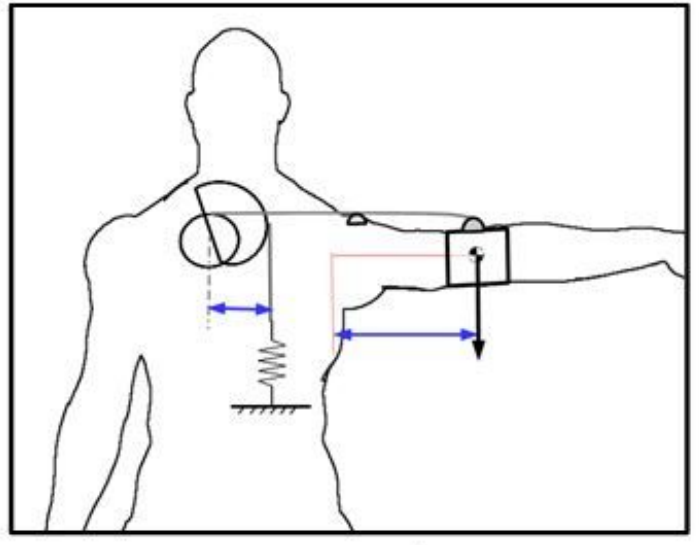

$\alpha=90^{\circ}$

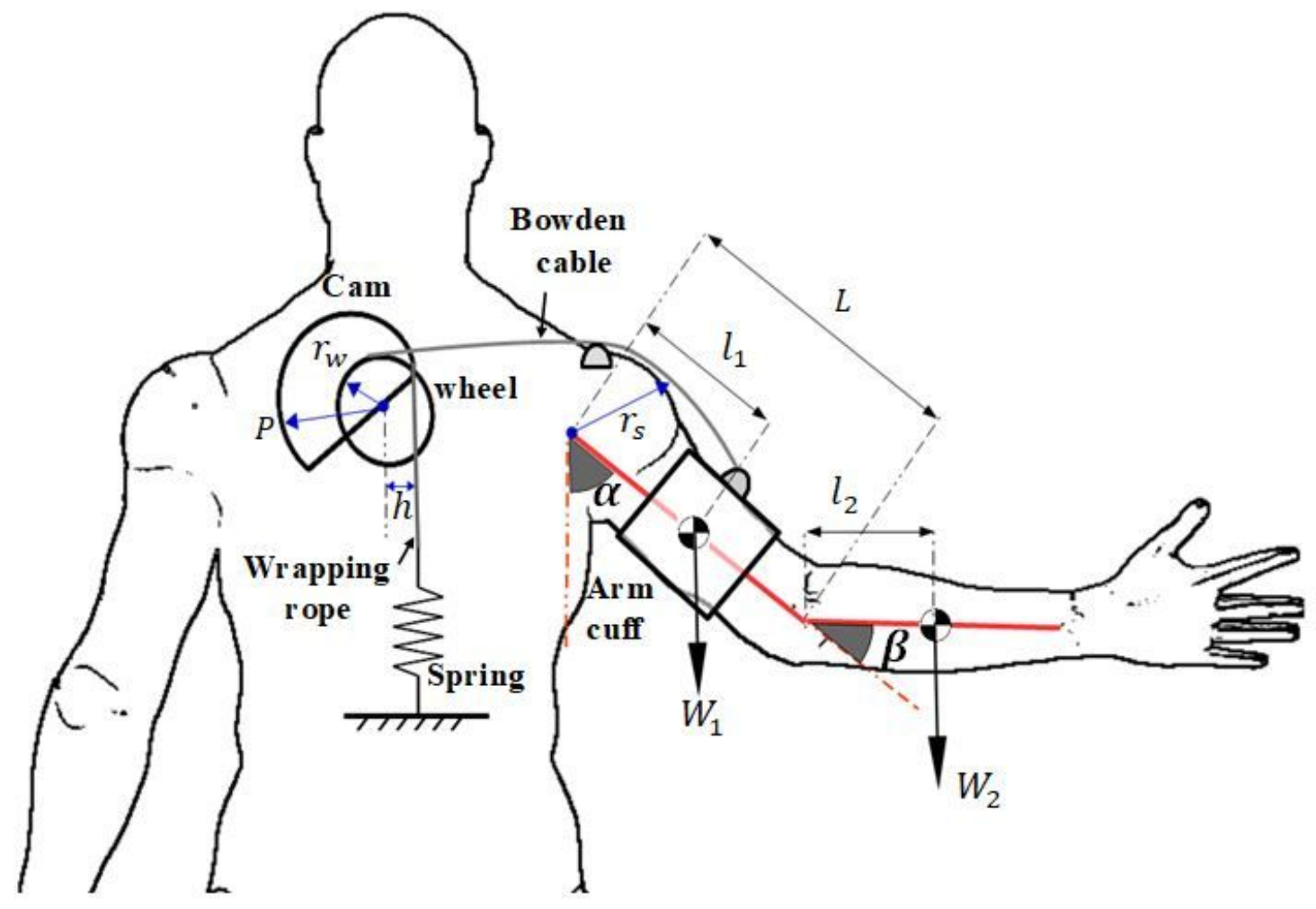

Figure 1

Schematic diagram of shoulder exoskeleton system concept (posterior view on right arm). Upper left and right figures represent two extreme configurations for shoulder elevation in the frontal plane. As the shoulder elevates from the $0^{\circ}$ (upper left figure) to $90^{\circ}$ (upper right figure), the cam-wheel rotates and adjust its effective moment arm relative to the wrapping rope to modulate the Bowden cable pulling force. 


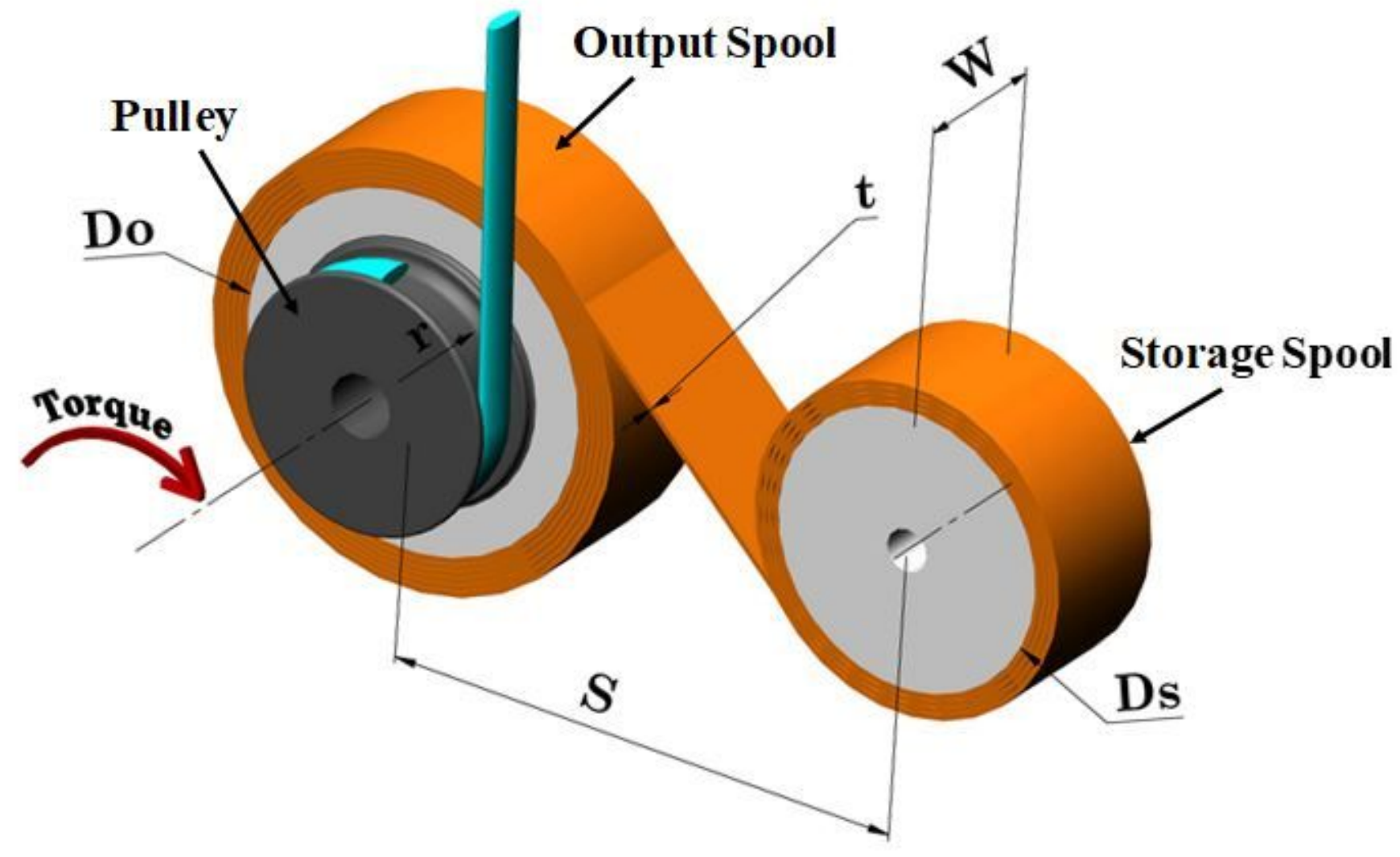

Figure 2

Schematic view of a constant-torque spring. The geometrical properties of the selected spring assembly are listed in the table. 


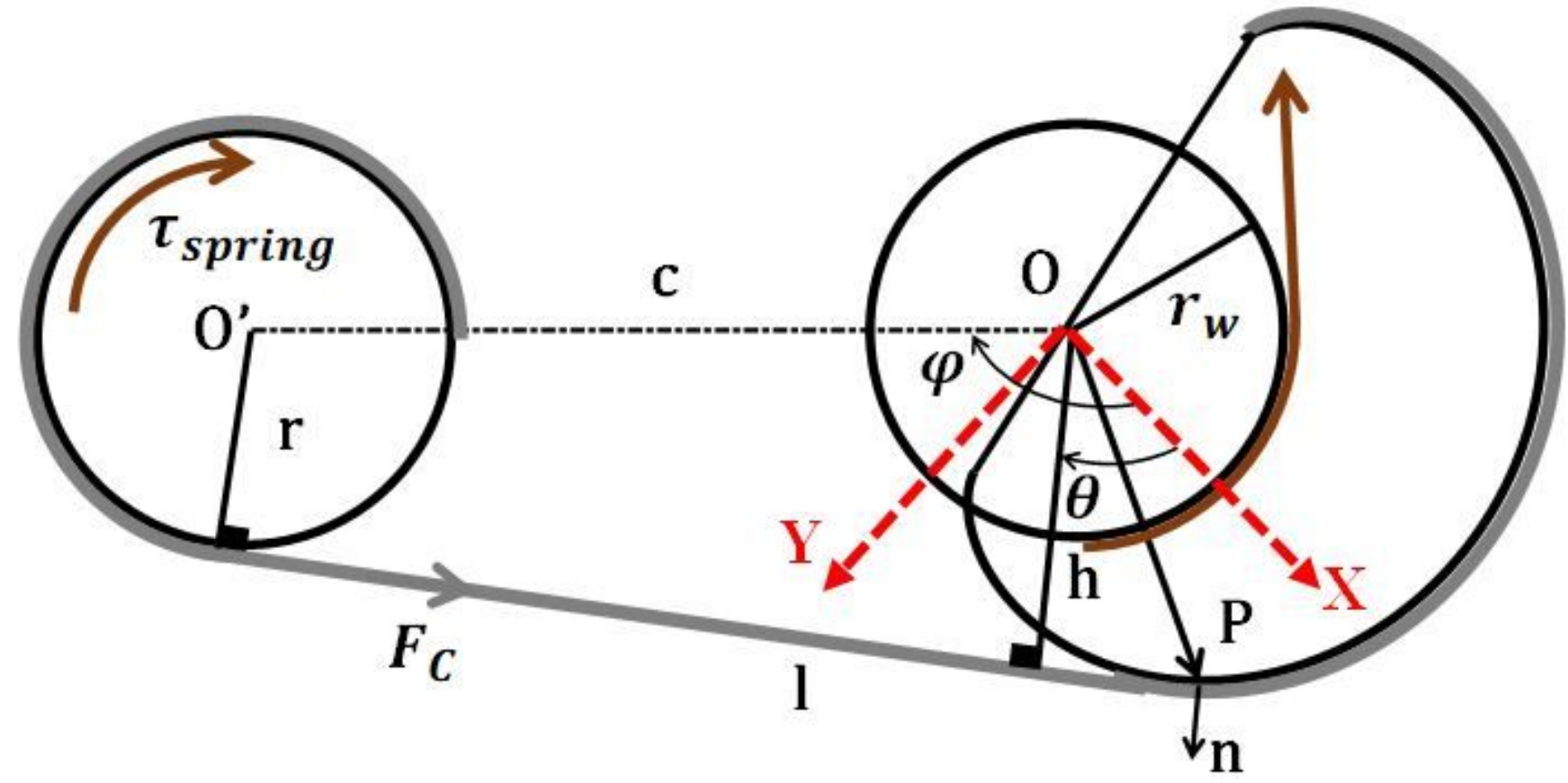

Figure 3

Schematic representation of the spring-cam-wheel module. 
A

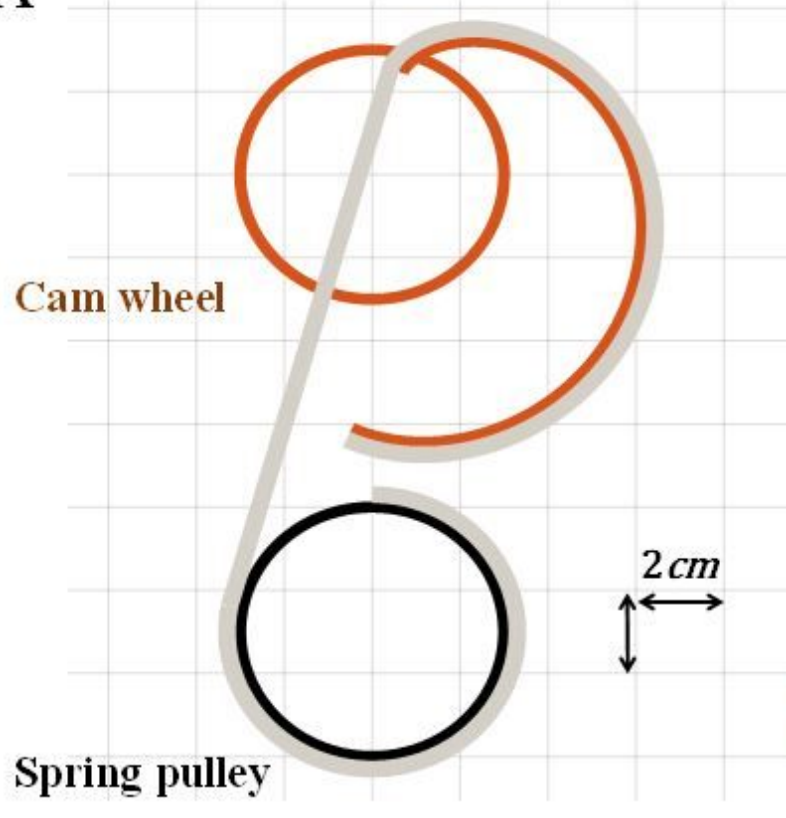

B

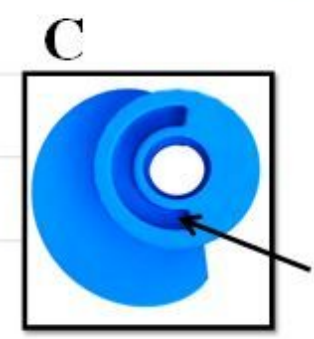

Stopper groove

D

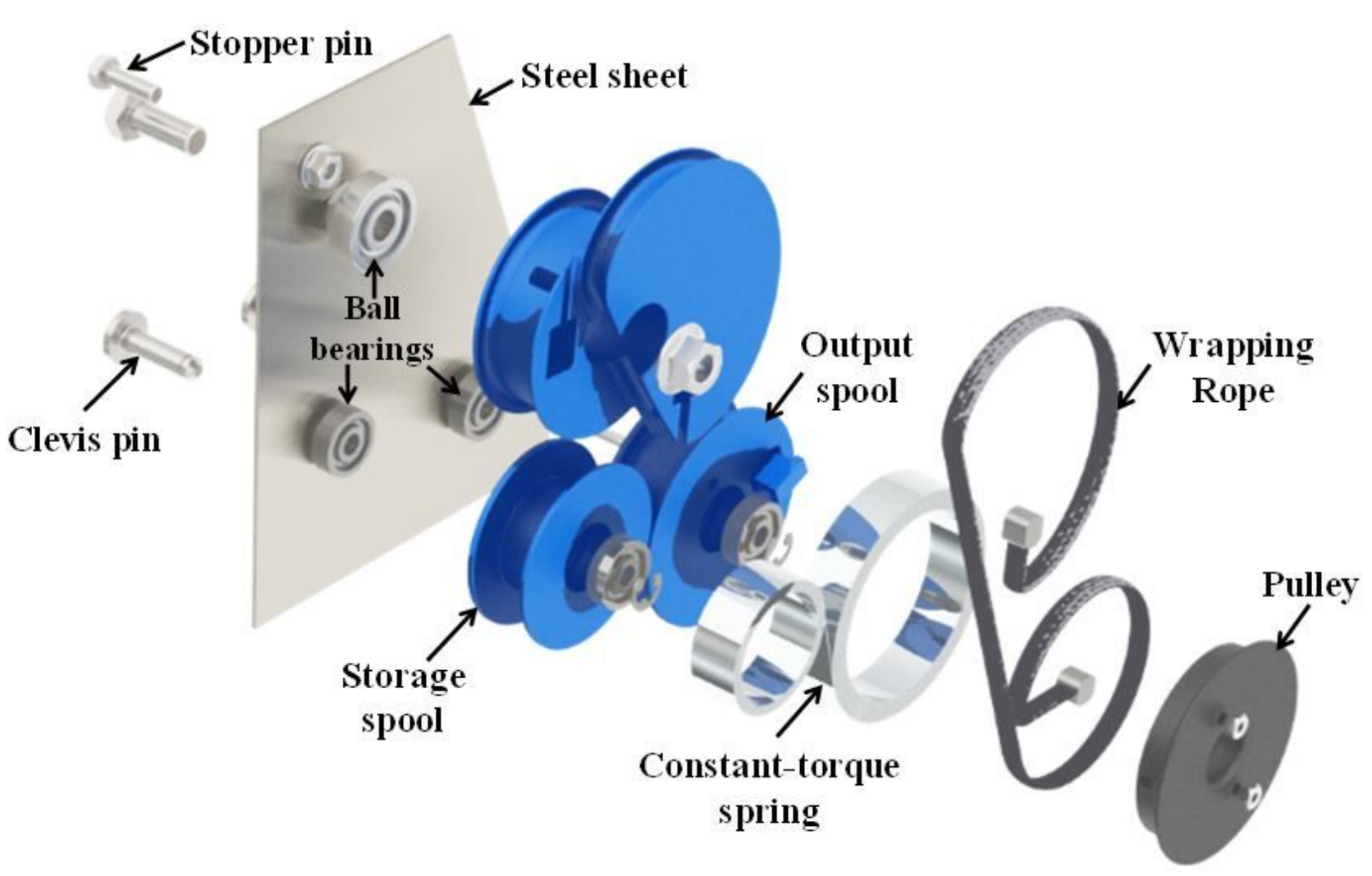

Figure 4

A) Profile of the cam-wheel. B) CAD model of the cam-wheel. C) Posterior view of the cam-wheel. D) Exploded view of the spring-cam-wheel module assembly. 


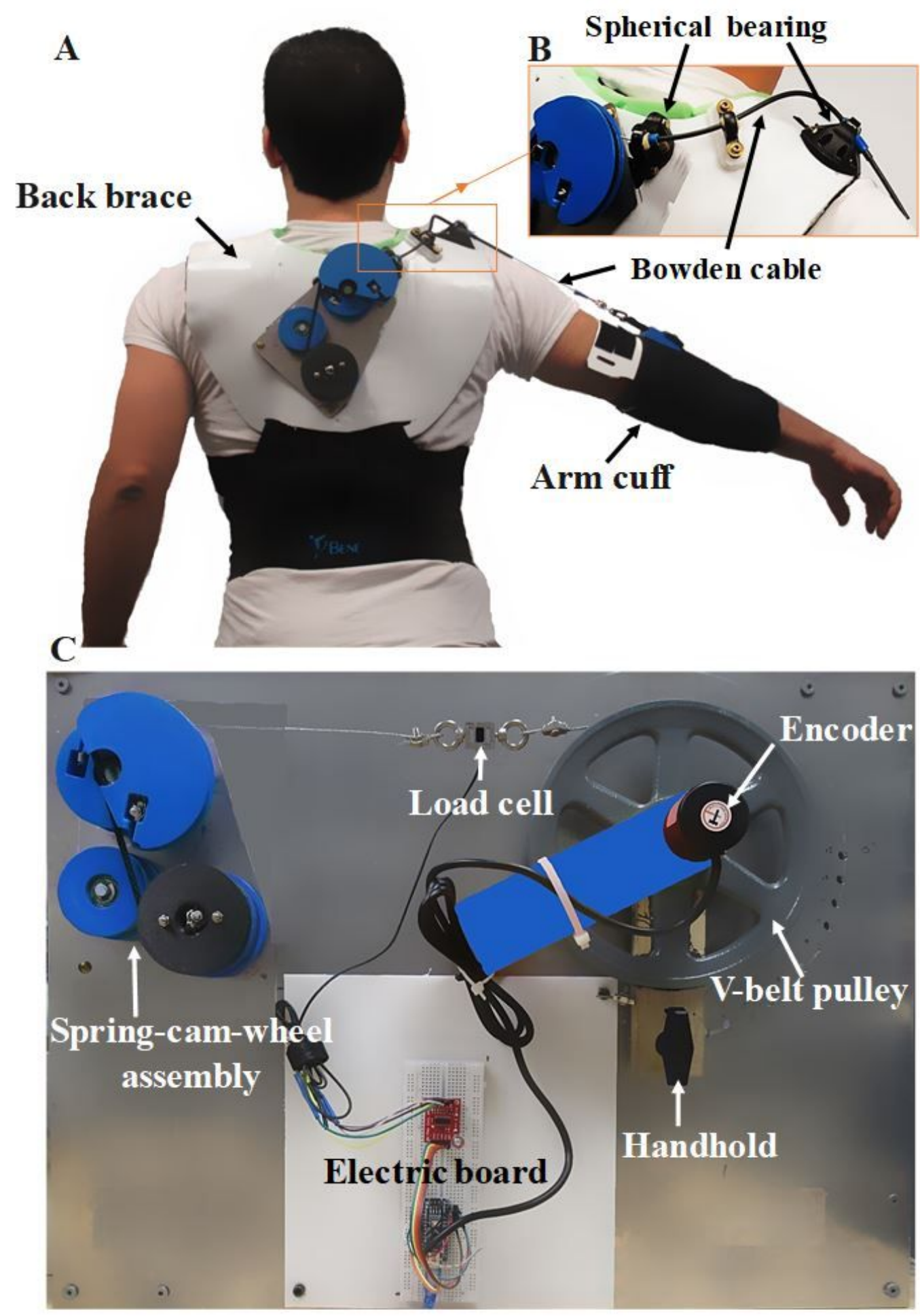

Figure 5

A) WPCSE prototype. B) Bowden cable routing path over the shoulder. C) Benchtop prototype. 

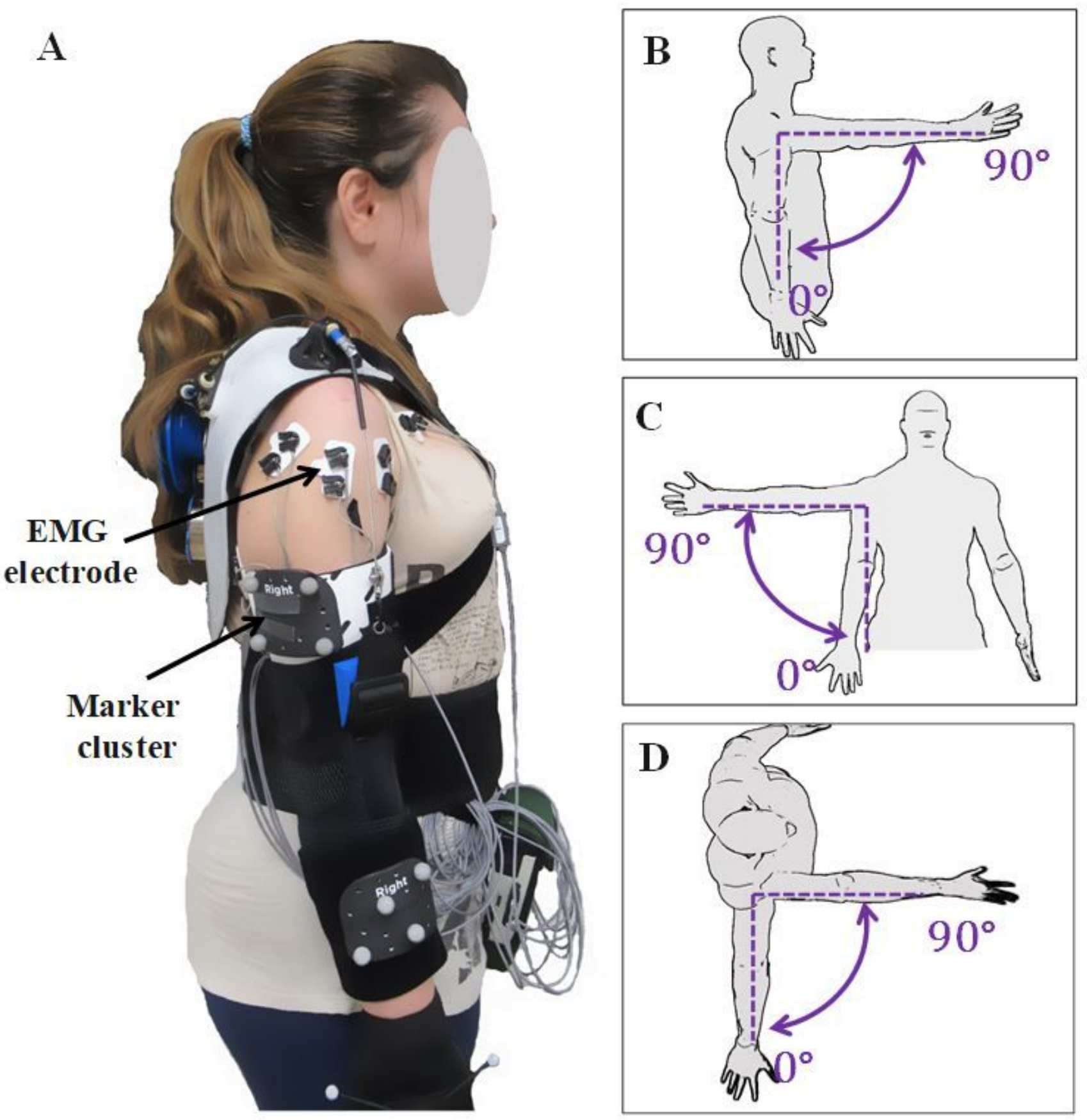

Figure 6

A) A participant with WPCSE, EMG electrodes, and reflective marker clusters. B). Shoulder elevation in the sagittal plane. C) Shoulder elevation in the frontal plane. D) Shoulder adduction/abduction in the horizontal plane. 


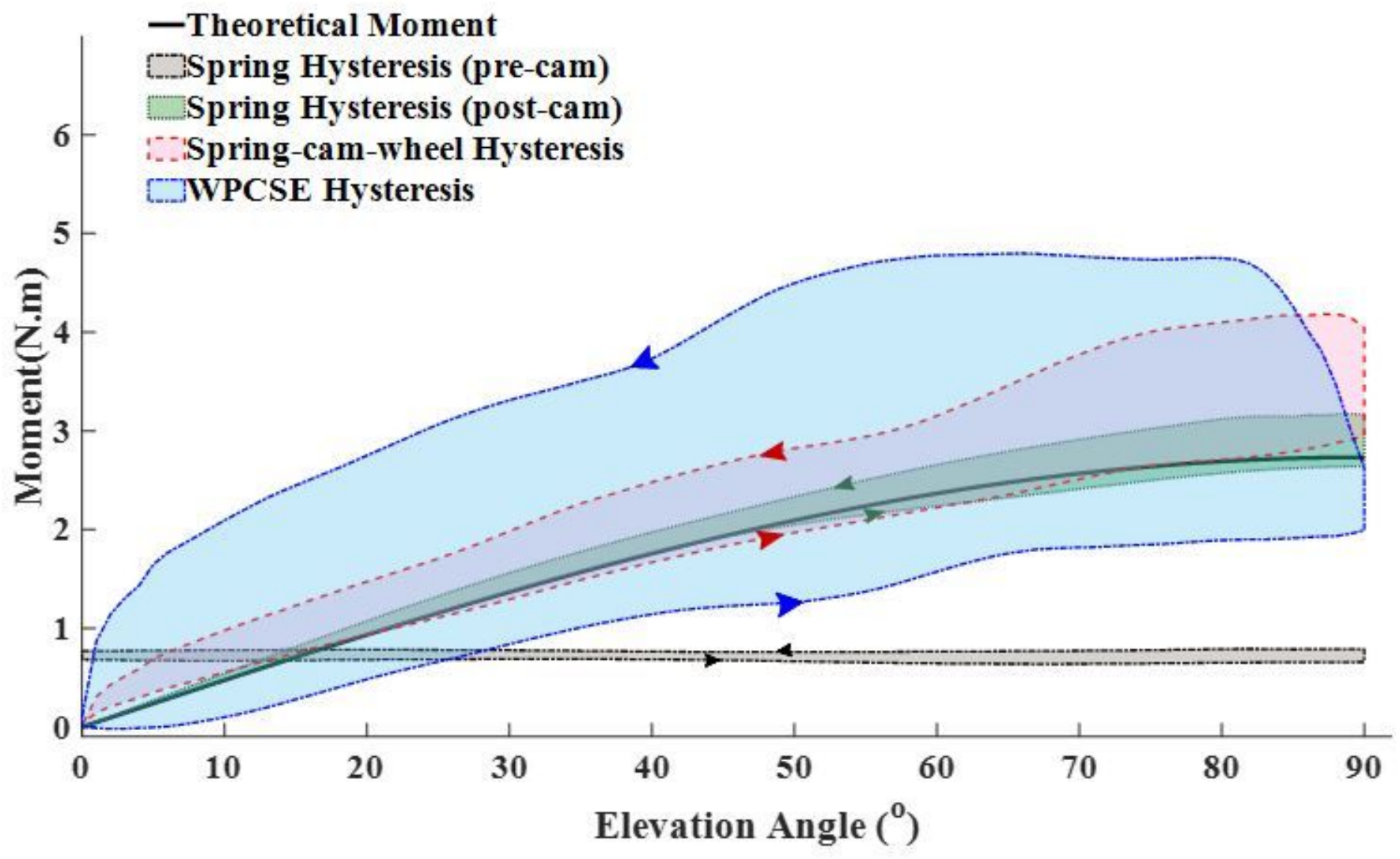

Figure 7

Joint moment produced by the constant-torque spring (pre-and post-cam), spring-cam-wheel module, and WPCSE during simulated shoulder elevation. The shaded regions indicate the degree of hysteresis, which is the difference in torque or moment between the positive and negative shoulder elevation phases. 


\section{Positive Elevation/Abduction}

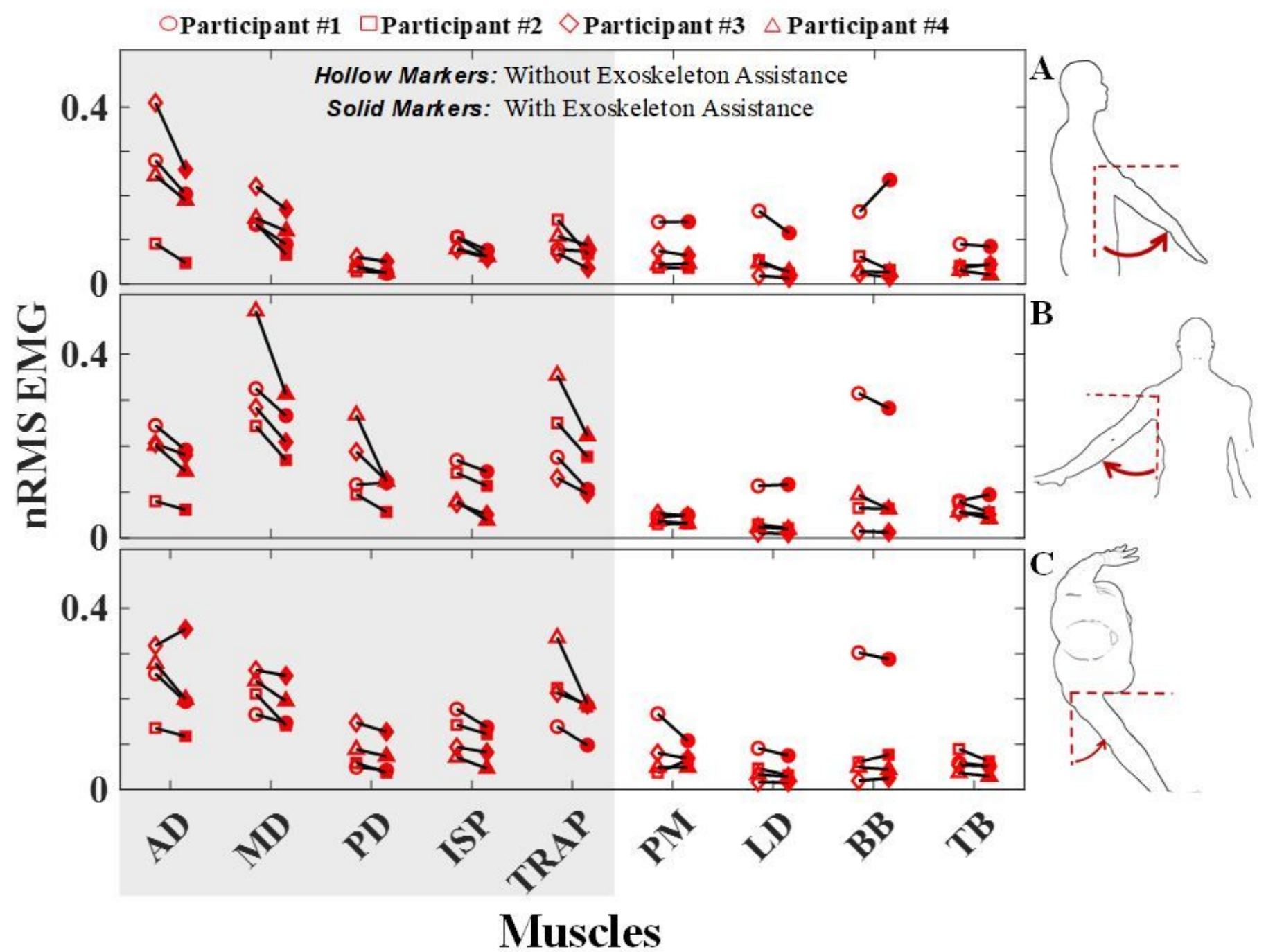

Figure 8

Root mean square of the normalized EMG (nRMS EMG) of several muscles crossing the shoulder during positive shoulder elevation in the sagittal plane (A), shoulder elevation in the frontal plane (B), and horizontal adduction (C) with and without exoskeleton assistance. Each marker shows the mean nRMS EMG across movement repetitions for each subject. Hollow and solid markers indicate trials without and with exoskeleton assistance, respectively. Shaded area shows muscles that primarily contribute force in the direction of shoulder elevation, based on their moment arms. 


\section{Negative Elevation/Abduction}

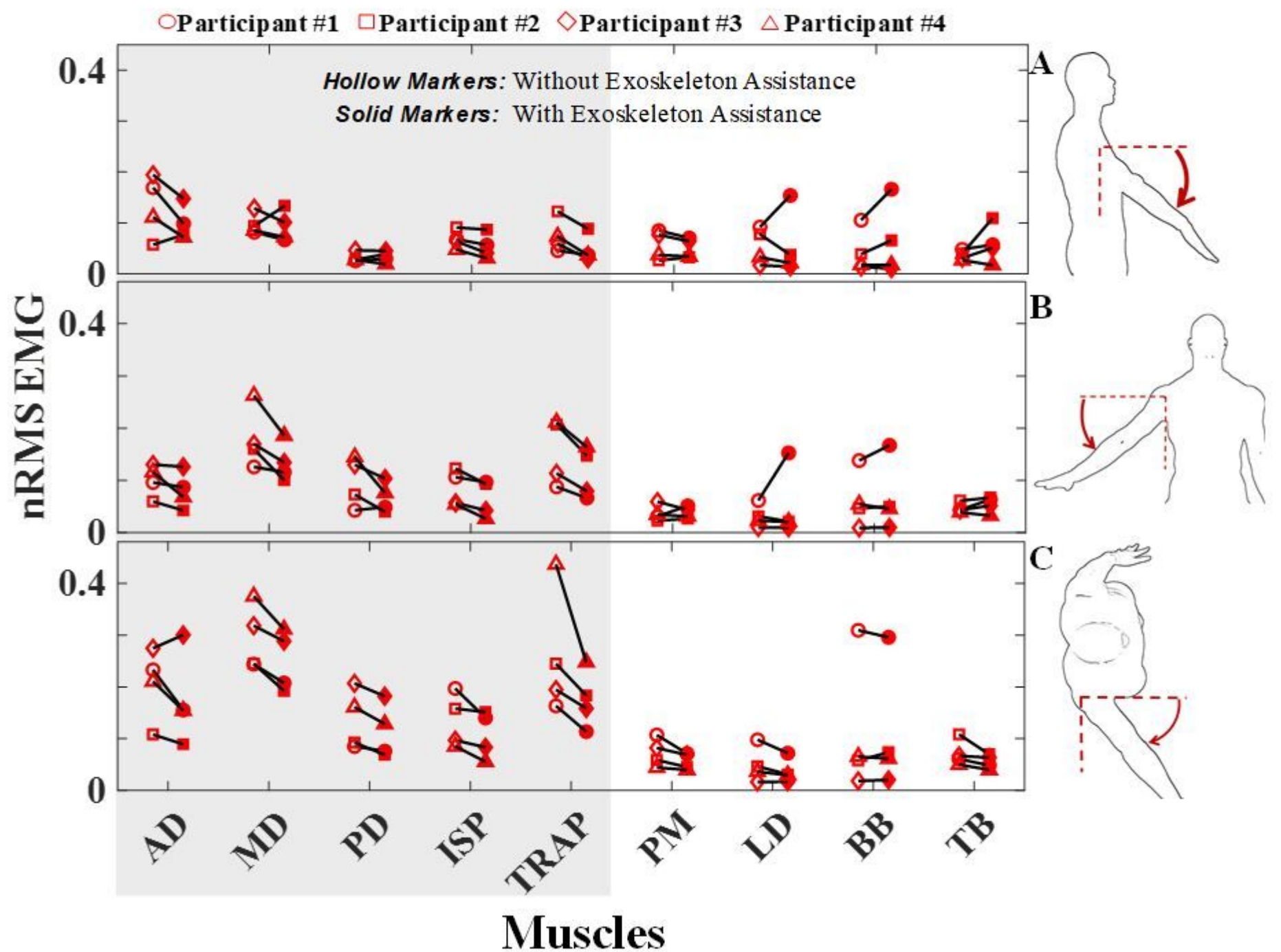

Figure 9

Root mean square of the normalized EMG (nRMS EMG) of several muscles crossing the shoulder during negative shoulder elevation in the sagittal plane (A), negative shoulder elevation in the frontal plane (B), and horizontal abduction (C) with and without exoskeleton assistance. Each marker shows the mean nRMS EMG across movement repetitions for each subject. Hollow and solid markers indicate trials without and with exoskeleton assistance, respectively. Shaded area shows muscles that primarily contribute force in the direction of shoulder elevation, based on their moment arms. 


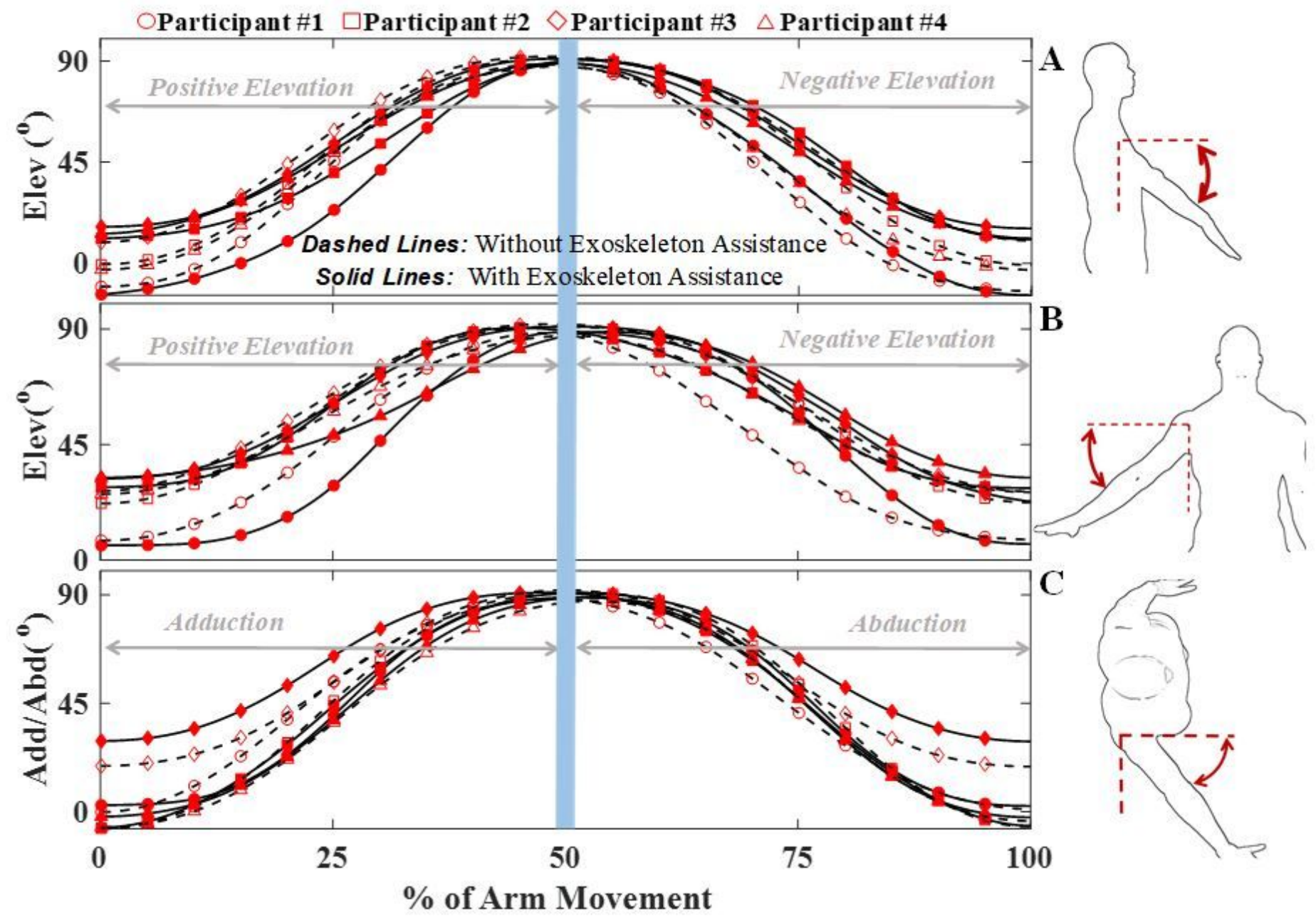

Figure 10

Shoulder angular displacement during elevation in the sagittal plane (A), elevation in the frontal plane (B), and horizontal adduction/abduction (C) with and without exoskeleton assistance. Each curve shows the mean of dominant angular displacement across movement repetitions for each subject. Hollow markers with dashed lines indicate trials without exoskeleton assistance, and solid markers with solid lines indicate trials with the exoskeleton assistance. Blue bar shows the approximate location of the upper limit of shoulder movements.

\section{Supplementary Files}

This is a list of supplementary files associated with this preprint. Click to download.

- supplementary.docx 